\title{
Effect of Straight, Inclined and Curved Fins on Natural Convection and Entropy Generation of a Nanofluid in a Square Cavity Influenced by a Magnetic Field
}

\author{
Yacine Khetib 1,2,*(D), Ahmad Aziz Alahmadi ${ }^{3}$, Ali Alzaed ${ }^{4}$, Hamidreza Azimy ${ }^{5}$, Mohsen Sharifpur ${ }^{6,7}, * \mathbb{D}$ \\ and Goshtasp Cheraghian $8, *$ (D)
}

check for

updates

Citation: Khetib, Y.; Alahmadi, A.A.; Alzaed, A.; Azimy, H.; Sharifpur, M.; Cheraghian, G. Effect of Straight,

Inclined and Curved Fins on Natural Convection and Entropy Generation of a Nanofluid in a Square Cavity Influenced by a Magnetic Field. Processes 2021, 9, 1339. https:// doi.org/10.3390/pr9081339

Academic Editors: Blaž Likozar

Received: 5 July 2021

Accepted: 28 July 2021

Published: 30 July 2021

Publisher's Note: MDPI stays neutral with regard to jurisdictional claims in published maps and institutional affiliations.

Copyright: (c) 2021 by the authors. Licensee MDPI, Basel, Switzerland. This article is an open access article distributed under the terms and conditions of the Creative Commons Attribution (CC BY) license (https:// creativecommons.org/licenses/by/ $4.0 /)$.
1 Mechanical Engineering Department, Faculty of Engineering, King Abdulaziz University, Jeddah 80204, Saudi Arabia

2 Center Excellence of Renewable Energy and Power, King Abdulaziz University, Jeddah 80204, Saudi Arabia

3 Department of Electrical Engineering, College of Engineering, Taif University, Taif 21944, Saudi Arabia; aziz@tu.edu.sa

4 Architectural Engineering Department, Faculty of Engineering, Taif University, Taif 21944, Saudi Arabia; alzaed@tu.edu.sa

Independent Researcher, 20100 Milan, Italy; hamidrezaazimy@yahoo.com

6 Department of Mechanical and Aeronautical Engineering, University of Pretoria, Pretoria 0002, South Africa

7 Department of Medical Research, China Medical University Hospital, China Medical University, Taichung 404, Taiwan

8 Independent Researcher, 38106 Braunschweig, Germany

* Correspondence: ykhetib@yahoo.com (Y.K.); mohsen.sharifpur@up.ac.za (M.S.); g.cheraghian@tu-braunschweig.de (G.C.)

Abstract: In this paper, the free convective heat transfer of nanofluids in a square cavity is simulated using a numerical method. The angle of the cavity could be changed in the horizontal axis from 0 to 90 degrees. The cavity is exposed under a constant magnetic field. Two opposite walls of the cavity are cold and warm, and the rest of the walls are insulated. On the hot wall, there are two fins with the same wall temperature. The equations were discretized by the finite volume method (FVM) and then solved using the SIMPLE algorithm. Three different fin configurations (straight, inclined and curved) were studied in terms of heat transfer rate and generation of entropy. According to the simulation results, the heat transfer rate was improved by tilting the fins toward the top or bottom of the cavity. At $\mathrm{Ra}=10^{5}$ and $\mathrm{Ha}=20$, the maximum heat transfer rate was achieved at a cavity inclination of $90^{\circ}$ and $45^{\circ}$, respectively, for straight and curved fins. In the horizontal cavity, heat transfer rate could be improved up to $6.4 \%$ by tilting the fins and up to $4.9 \%$ by warping them. Increasing the Hartmann number from 0 to 40 reduced the Nusselt number and entropy generation by $37.9 \%$ and $33.8 \%$, respectively.

Keywords: natural convection; straight fin; inclined fin; curved fin; magnetic field

\section{Introduction}

Natural convection heat transfer has extensive applications in several industries. This type of heat transfer occurs without any force applied by a fan or similar equipment, for example, in ovens and furnaces. Heat transfer by natural convection in closed enclosures has been used in various industries, such as aerospace and solar industries [1,2]. Accordingly, there has been a growing interest in this type of enclosure in recent years [3-5]. Additionally, numerous studies have been recently conducted on nanofluids in closed enclosures as an attractive research area.

Nanoparticles have many applications, and researchers have used nanoparticles in various fields, including solar, heat exchangers, medicine, etc. [6-8]. The enhancement of heat transfer rate (HTR) by adding nanofluids is one motivation behind these studies. Several types of research have focused on the HTR of nanofluids [9-17]. 
Fins can be used to improve HTR in cavities by increasing heat transfer surface area. The effectiveness of fins has been confirmed in forced convection. However, due to reduced velocity in natural convection, the size and shape of the fin determine whether it improves or diminishes HTR, particularly in cavities [18-24]. Alnaqi et al. [25] placed a fin of constant thermal conductivity in an inclined square cavity to investigate its effect on natural convection heat transfer. Their results indicated an intensification in the Nusselt number $(\mathrm{Nu})$ with the Rayleigh number $(\mathrm{R} a)$. Alrashed [26] compared the effect of two fins with different inclinations (angles) on the HTR. The fins with adjustable inclinations were placed on the sides of the cavity. This study revealed the significant impact of fin inclination on HTR in the cavity.

Magnetic fields may also influence HTR. According to the literature on forced convection, HTR may be enhanced by applying a magnetic field [27]. In contrast, natural convection heat transfer may be diminished by applying a magnetic field due to a reduction in the fluid velocity [28-37]. For example, Pordanjani et al. [38] considered the effect of fins on HTR in an enclosure under a magnetic field of different intensities. According to their results, the magnetic field reduced HTR by decreasing fluid velocity. The HTR further decreased at higher field intensities.

Generation of entropy (GOE) can be calculated as a better way of studying the efficiency of thermal equipment. GOE is a measure of irreversibility and energy loss in the system. Accordingly, in addition to HTR, GOE has also been investigated in numerous studies [39-45]. Zhou et al. [46] studied HTR and GOE in a nanofluid-filled cavity at a Grashof number $(\mathrm{Ga})$ of $10^{4}-10^{6}$ and a nanoparticle volume fraction of $0.01-0.15 \%$. They found an increase in HTR and local GOE with an increasing Ga and nanoparticle volume fraction. Dutta et al. [47] investigated GOE and HTR of the $\mathrm{Cu}$-water nanofluid in a rhombic enclosure exposed to a magnetic field. Their results showed a decrease in GOE with an increasing Hartmann number $(\mathrm{Ha})$ at all Rayleigh number $(\mathrm{Ra})$ values and cavity inclinations.

In another study, $\mathrm{Li}$ et al. [44] examined the free convection of nanofluids in a cavity with entropy generation despite a circular barrier in the center of the cavity. Their findings showed that increasing $R a$ could increase $N u$ by 4.5 times, but increasing the Hartmann number reduced GOE by $35 \%$.

Fin-equipped cavities are widely used in the industry, including in furnaces and kitchen ovens. HTR may be improved by adding a nanofluid to the cavity. However, it should be noted that this can have different impacts on natural convection heat transfer due to increased viscosity. The flow in the cavity can be controlled by a magnetic field. Fins with different geometries and inclinations can significantly affect heat transfer in cavities. This study compares the effect of three different fins on heat transfer and GOE in a square cavity exposed to a magnetic field. The cavity was filled with a nanofluid and can assume different inclinations relative to the horizon. Two fins were installed on the hot wall of the cavity, and the effect of straight, inclined and curved fins was studied. As an innovative aspect of this study, the impact of the curved fin on the flow and temperature fields was investigated. GOE was also investigated by adjusting the fin inclination, curvature and cavity inclination.

\section{Problem Description}

A 2D square cavity with unit length filled with a $\mathrm{Al}_{2} \mathrm{O}_{3}$-water nanofluid was studied. The cavity can tilt and make an angle of $\gamma$ with the horizon. A constant, horizontal magnetic field was applied to the cavity. The right and left walls were respectively maintained at constant temperatures of $T_{c}$ and $T_{H}$. Moreover, the top and bottom sides were insulated. Two fins were installed on the hot wall at the same temperature as the wall. As illustrated by Figure 1, the fins installed may be straight, inclined or curved. Natural convection was established in the cavity by Boussinesq approximation. 


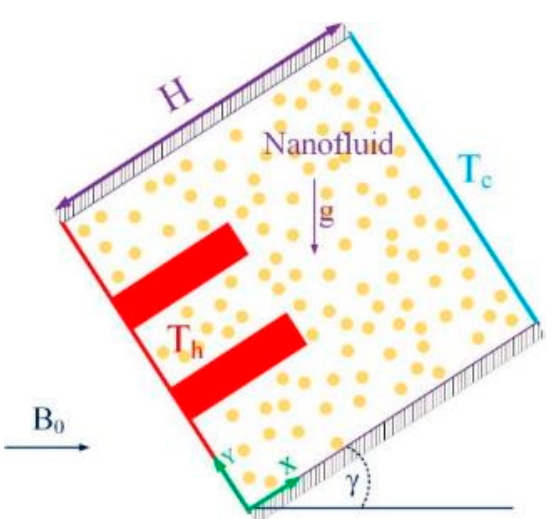

(a) Straight Fins

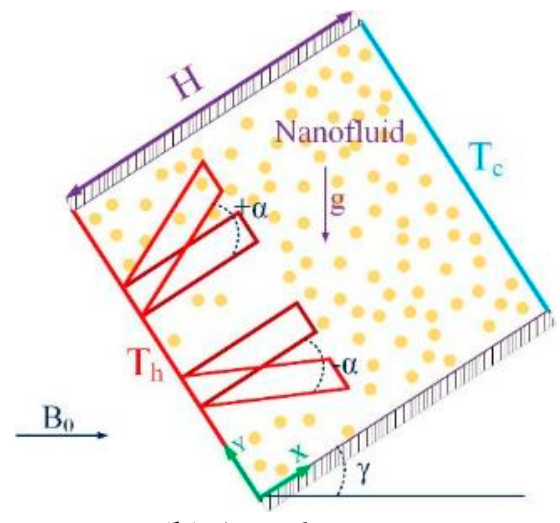

(b) Angular Fins

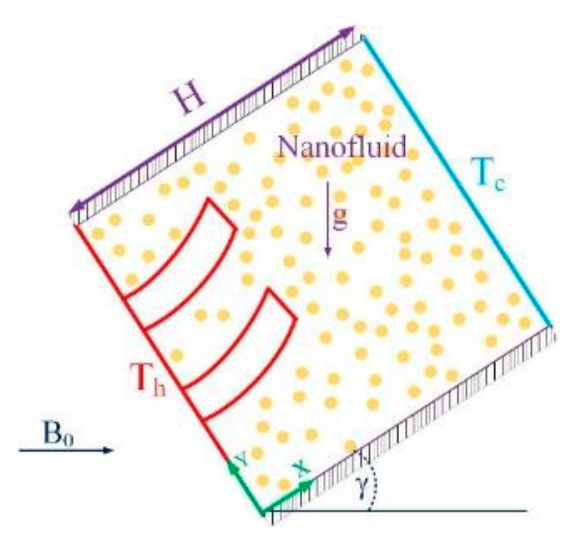

(c) Curve Fins

Figure 1. The geometry of the cavity and different fins.

\subsection{Governing Equations and Boundary Condition}

The equations governing the nanofluid flow are given below. The equations with the indices $a$ and $b$ are, respectively, dimensional and dimensionless. These equations include conservations of mass, momentum, and energy [38,44].

$$
\begin{gathered}
u_{x}+v_{y}=0 \\
U_{X}+V_{Y}=0 \\
u u_{x}+v u_{y}=-\frac{1}{\rho_{n f}} p_{x}+\frac{\mu_{n f}}{\rho_{n f}}\left(u_{x x}+u_{y y}\right)+g \beta_{n f}\left(T-T_{c}\right) \sin \gamma \\
U U_{X}+V U_{Y}=-P_{X}+\frac{\mu_{n f}}{\rho_{n f} \alpha_{f}}\left(U_{X X}+U_{Y Y}\right)+\frac{\beta_{n f}}{\beta_{f}} R a \operatorname{Pr} \theta \sin \gamma \\
u v_{x}+v v_{y}=-\frac{1}{\rho_{n f}} p_{y}+\frac{\mu_{n f}}{\rho_{n f}}\left(v_{x x}+v_{y y}\right)+g \beta_{n f}\left(T-T_{c}\right) \cos \gamma-\frac{\sigma_{n f} B_{0}^{2}}{\rho_{n f}} v \\
U V_{X}+V V_{Y}=-P_{Y}+\frac{\mu_{n f}}{\rho_{n f} \alpha_{f}}\left(V_{X X}+V_{Y Y}\right)+\frac{\beta_{n f}}{\beta_{f}} R a \operatorname{Pr} \theta \cos \gamma+\frac{\rho_{f}}{\rho_{n f}} \frac{\sigma_{n f}}{\sigma_{f}} \operatorname{PrHa}^{2}(-V) \\
u T_{x}+v T_{y}=\alpha_{n f}\left(T_{x x}+T_{y y}\right) \\
U \theta_{X}+V \theta_{X}=\frac{\alpha_{n f}}{\alpha_{f}}\left(\theta_{X X}+\theta_{Y Y}\right)
\end{gathered}
$$

The equations with the indices $a$ were nondimensionalized using the following relations and transformed to those with the index $b[36,39]$.

$$
\begin{gathered}
X=\frac{x}{l}, Y=\frac{y}{l}, U=\frac{u l}{\alpha_{f}}, V=\frac{v l}{\alpha_{f}}, P=\frac{p l^{2}}{\rho_{n f} \alpha_{f}^{2}}, \theta=\frac{T-T_{c}}{T_{h}-T_{c}} \\
P r=\frac{\vartheta_{f}}{\alpha_{f}}, R a=\frac{g \beta_{f} l^{3}\left(T_{h}-T_{c}\right) P r}{\vartheta_{f}^{2}}, H a^{2}=\frac{\sigma_{f} B_{0}^{2} l^{2}}{\mu_{f}}
\end{gathered}
$$

According to the dimensionless Equation (5), the dimensionless boundary conditions were obtained, as shown in Figure 2. 


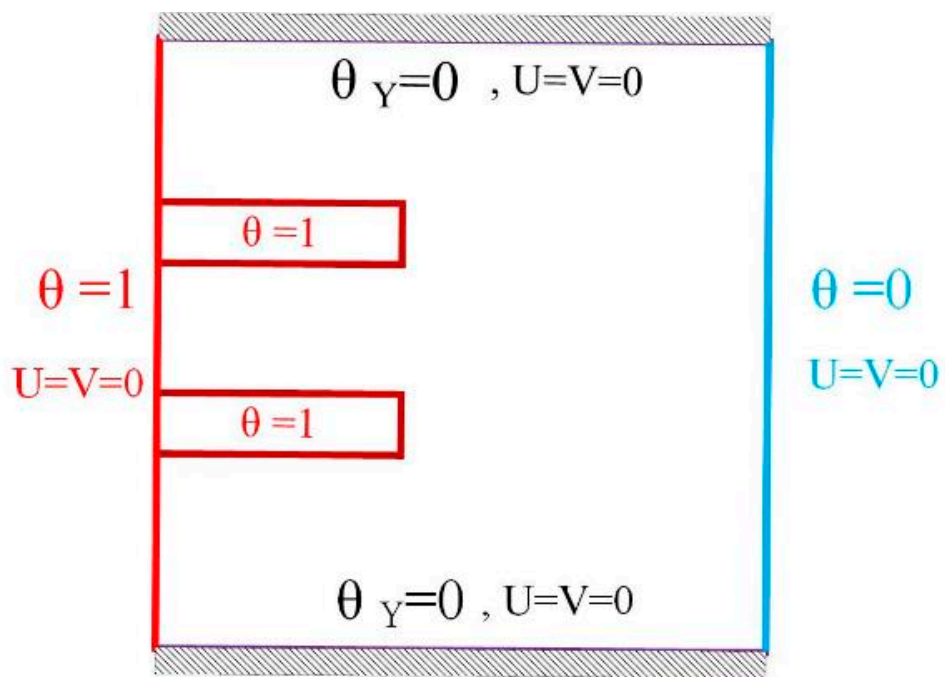

Figure 2. The dimensionless boundary conditions.

\subsection{Auxiliary Equations}

GOE can be calculated from the following dimensionless equation [36,39].

$$
S_{g e n}=\frac{k_{n f}}{k_{f}}\left(\left(\frac{\partial \theta}{\partial X}\right)^{2}+\left(\frac{\partial \theta}{\partial Y}\right)^{2}\right)+\zeta\left\{2\left[\left(\frac{\partial U}{\partial X}\right)^{2}+\left(\frac{\partial V}{\partial Y}\right)^{2}\right]+\left(\frac{\partial U}{\partial Y}+\frac{\partial V}{\partial X}\right)^{2}\right\}+\zeta \frac{\sigma_{n f}}{\sigma_{f}} \frac{\mu_{f}}{\mu_{n f}} H a^{2} V^{2}
$$

where $\zeta=\frac{\mu_{n f} T_{0}}{k_{f}}\left(\frac{\alpha_{f}}{L\left(T_{h}-T_{C}\right)}\right)^{2}$. Further, the total GOE is expressed as follows:

$$
S_{G, T}=\int S_{g e n} d \Omega=\iint_{0}^{1} S_{g e n} d X d Y
$$

The Nusselt number $(\mathrm{Nu})$ shows the extent of heat transfer and is calculated as follows:

$$
\begin{gathered}
N u_{Y}=\frac{h L}{k_{f}} \\
h=\frac{q_{\omega}}{T_{h}-T_{c}} \\
q_{\omega}=k_{n f}\left(\frac{\partial T}{\partial X}\right) \\
N u=-\frac{k_{n f}}{k_{f}}\left(\frac{\partial \theta}{\partial X}\right)
\end{gathered}
$$

Moreover, the average $\mathrm{Nu}$ is obtained from the following equation.

$$
N u_{M}=\frac{1}{L} \int_{0}^{L} N u_{Y} d Y=-\frac{1}{L} \frac{k_{n f}}{k_{f}} \int_{0}^{L}\left(\frac{\partial \theta}{\partial X}\right)_{Y} d Y
$$

The relations used for measuring the nanofluid properties are presented below [36].

$$
\begin{aligned}
& \sigma_{n f}=(1-\varphi) \sigma_{f}+\varphi \sigma_{s} \\
& \rho_{n f}=(1-\varphi) \rho_{f}+\varphi \rho_{s}
\end{aligned}
$$




$$
\begin{gathered}
(\rho \beta)_{n f}=(1-\varphi)(\rho \beta)_{f}+\varphi(\rho \beta)_{s} \\
\left(\rho c_{p}\right)_{n f}=(1-\varphi)\left(\rho c_{p}\right)_{f}+\varphi\left(\rho c_{p}\right)_{s} \\
\alpha_{n f}=\frac{k_{n f}}{\left(\rho c_{p}\right)_{n f}}
\end{gathered}
$$

Various relations have been presented in the literature [48] for measuring the thermal conductivity and viscosity of nanofluids. This model considers the Brownian motion of nanoparticles for better simulation of the thermal conductivity and viscosity. The Brownian motion of nanoparticles in nanofluids refers to their random continuous motion in the fluid induced by collisions with liquid molecules, leading to dispersion of nanoparticles in the fluid [49].

$$
\begin{aligned}
\frac{k_{n f}}{k_{f}}=0.991+ & 0.276 T \varphi+77.6 \varphi^{2}+3641.231 T \varphi^{2}+\frac{0.00217}{\sin (T-\varphi)}-6.01 \times 10^{-6} T^{2} \\
& -3647.099 T \varphi \sin (\varphi) \\
\mu_{n f}=\mu_{\text {Static }}+ & \mu_{\text {Brownian }}=\frac{\mu_{f}}{(1-\varphi)^{2.5}}+5 \times 10^{4} \beta \varphi \rho_{f}\left(C_{p}\right)_{f} \frac{\mu_{f}}{k_{f} P r} \sqrt{\frac{k_{f} T}{\rho_{p} d_{p}}} f(T, \varphi)
\end{aligned}
$$

The thermal model used in $[48,50]$ is depicted in Figure 3. Further, this study uses the viscosity models presented by Vajjha et al. [48] and Brinkman [51]. The values of $\beta$ and $f(T, \varphi)$ for the $\mathrm{Al}_{2} \mathrm{O}_{3}$-water nanofluid are given below [48].

$$
\begin{gathered}
f(T, \varphi)=\left(2.8217 \times 10^{-2} \varphi+3.917 \times 10^{-3}\right)\left(\frac{T}{T_{0}}\right)+\left(-3.0669 \times 10^{-2} \varphi-3.91123 \times 10^{-3}\right) \\
\beta=8.4407(100 \varphi)^{-1.07304}
\end{gathered}
$$

Table 1 presents the properties of water and $\mathrm{Al}_{2} \mathrm{O}_{3}$.

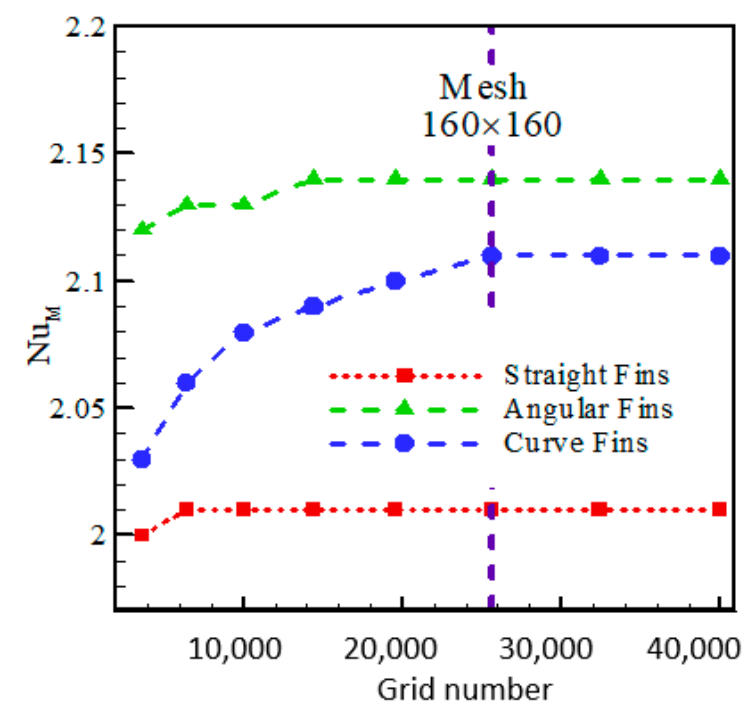

(a)

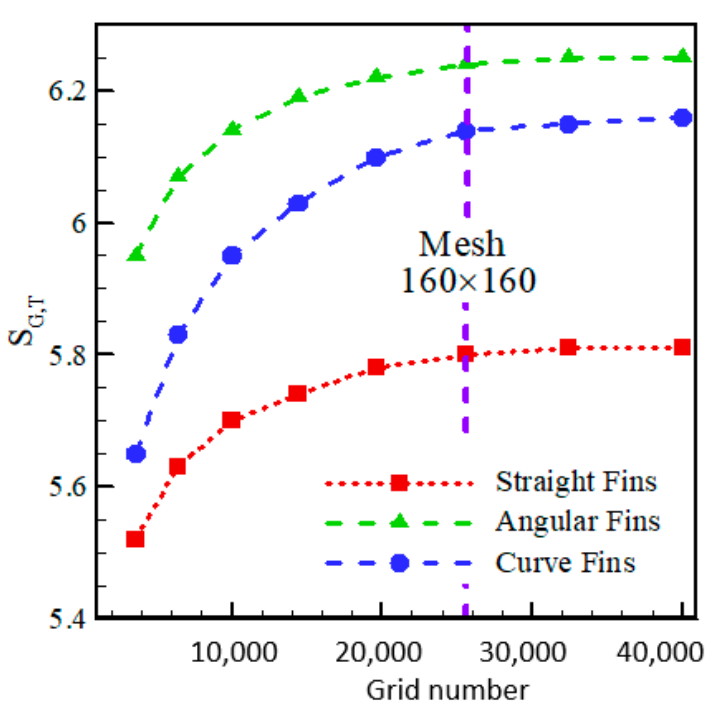

(b)

Figure 3. The variations of average $\mathrm{Nu}$ over the cold wall and GOE in the cavity for $\mathrm{Ra}=10^{5}, \mathrm{Ha}=20, \varphi=0.03, \gamma=0^{\circ}$ and different grid sizes. 
Table 1. The properties of water and nanoparticles.

\begin{tabular}{ccccccc}
\hline & $C_{P}(\mathrm{~J} / \mathrm{kg} \cdot \mathrm{K})$ & $\boldsymbol{k}(\mathrm{W} / \mathbf{m} \cdot \mathbf{K})$ & $\rho\left(\mathrm{kg} / \mathrm{m}^{3}\right)$ & $\mu(\mathrm{kg} / \mathrm{m} \cdot \mathbf{s})$ & $\sigma(\Omega \cdot \mathrm{m})^{-1}$ & $d_{S}(\mathrm{~nm})$ \\
\hline Water & 4179 & 0.613 & 997.1 & 0.001 & 0.05 & - \\
\hline $\mathrm{Al}_{2} \mathrm{O}_{3}$ & 765 & 40 & 3970 & - & $10^{-12}$ & 47 \\
\hline
\end{tabular}

\subsection{Numerical Procedure}

The equations were discretized before being solved by the finite volume method. The cavity geometry was meshed uniformly using identical quadrilateral elements. Next, a Fortran code was developed based on the SIMPLE algorithm. Each run took nearly $40 \mathrm{~min}$ on a computers with a 3.7 GHZ core i7 CPU and 16 GB DDR4 RAM. Further, the following convergence criterion was selected to terminate the solution.

$$
\Phi=\sum_{J} \sum_{I}\left|\frac{\varphi^{n+1}-\varphi^{n}}{\varphi^{n+1}}\right| \leq 10^{-8}
$$

\subsection{Grid Analysis}

To find the best grid size, the grid independence was investigated based on the average $N u$ over the cold wall and GOE at $R a=10^{5}, H a=20, \varphi=0.03, \gamma=0^{\circ}$ by changing the grid size from 3600 to 40,000. The variations of the average $N u$ and GOE for different grid sizes are shown in Figure 3. Eventually, a grid with 25,600 elements $(160 \times 160)$ was adopted.

\subsection{Validation}

First, the results from the developed model were compared with those reported by Oztop and Abu-Nada [52], who investigated a simple nanofluid-filled cavity with vertical hot and cold walls. According to Figure 4, which plots the average Nu versus nanoparticle volume fraction, there is a slight discrepancy between the results.

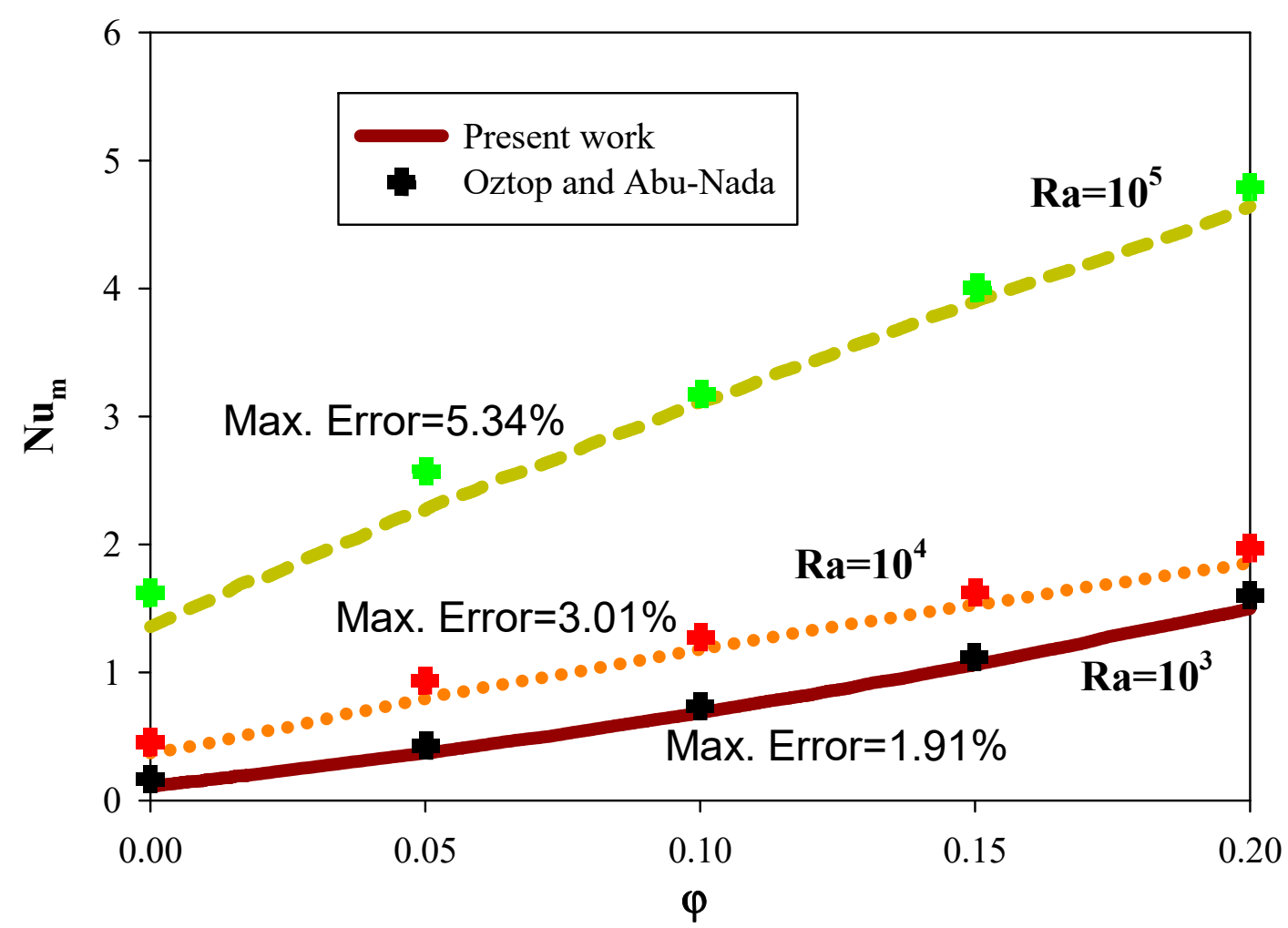

Figure 4. The performance of the model for natural convection in the nanofluid-filled cavity developed. 
Next, the model was evaluated for flow under a magnetic field. Accordingly, the model was compared with the results of Aminossadati et al. [53]. The average $\mathrm{Nu}$ for various $\mathrm{Nu}$ and $\mathrm{Ha}$ is indicative of the magnetic field are plotted in Figure 5. Obviously, the developed model exhibits excellent accuracy for natural convection in a nanofluid exposed to a magnetic field and, therefore, can be employed.

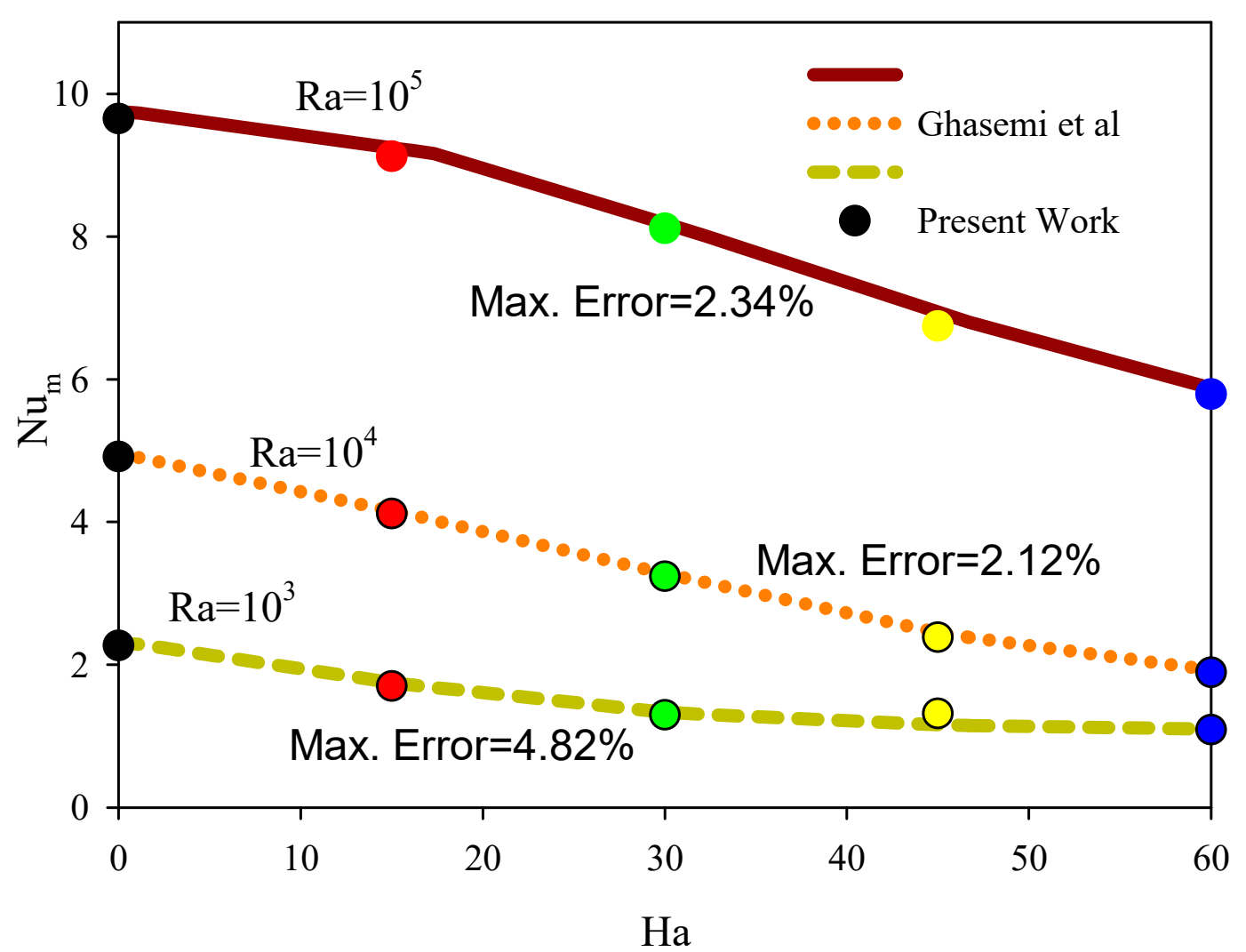

Figure 5. A comparison of the developed model with the results of previous works for a nanofluid exposed to a magnetic field.

According to the two comparisons made above, the highest error of the results of this article with previous articles is $5.34 \%$ and $4.82 \%$ for the results of Oztop and Abu-Nada [52] and Aminossadati et al. [53], respectively. From the above comparison, it was found that there is a slight difference between the results of the present work and the results of the references [52,53]. Therefore, the code used for this research has acceptable accuracy.

\section{Results and Discussion}

\subsection{Effect of Fin Inclination}

Figure 6 shows the (a) flow, (b) temperature and (c) entropy fields at different fin inclinations for the specified constants. Figure 6a shows that as the fins are angled, the number of stream functions increases at the center of the vortex. This can be caused by the tip of the fins approaching the walls and thus increasing the space in the middle of the cavity. Increasing the value in the center of the contours means increasing the maximum value of the stream function, which also means increasing the spin speed of the vortex. In all the above cases, the vortex rotates clockwise. Vortex penetration in the middle of the fins can lead to an increase in fluid collision with the wall in that area. In these areas, the rate of heat transfer also increases. In Figure $6 \mathrm{~b}$, in temperature counters, the presence of more constant temperature lines in an area means an increase in the temperature difference in that area. The highest density of constant temperature lines is in the two areas of the upper part of the right wall and the lower part of the left wall. In these areas, due to the direction of rotation of the vortex, the temperature difference is high, and the density of the lines is higher. The heat transfer rate is also high in these areas. When the fin is lower, line 
densities may occur on the fin tip, so heat transfer from the tip of the fin increases in this area. In Figure 6c, the appearance of entropy production lines in locally produced entropy contours means the formation of entropy in that area. By comparing the temperature and entropy contours produced, it can be seen that the density areas of the lines are similar in both. The dependence of the generated entropy on the temperature gradient has caused the density of the entropy field lines to be very similar to the temperature field. It can also be seen that with different variations of the fin angle, the amount of local entropy is always high in the lower corner of the lower fin. Increasing the angle of the fins causes the place of maximum entropy generation to be closer to the lower wall.

$\alpha$

(a) $\psi$

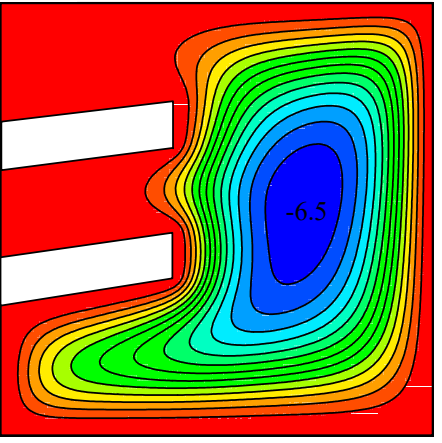

$-10$

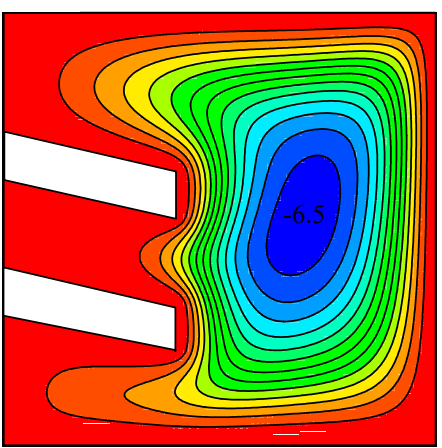

20

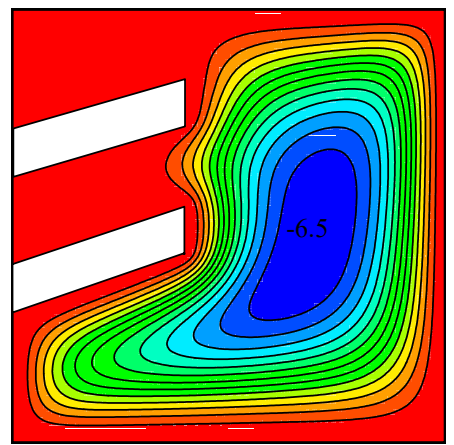

$-20$

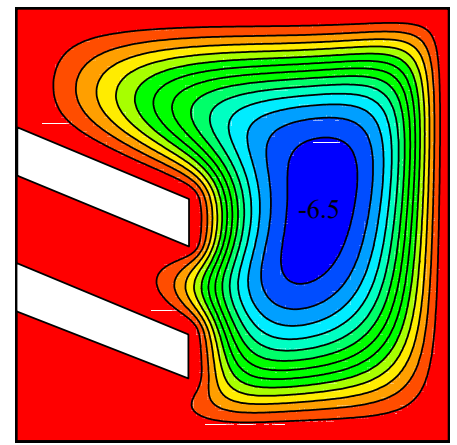

(b) $T$
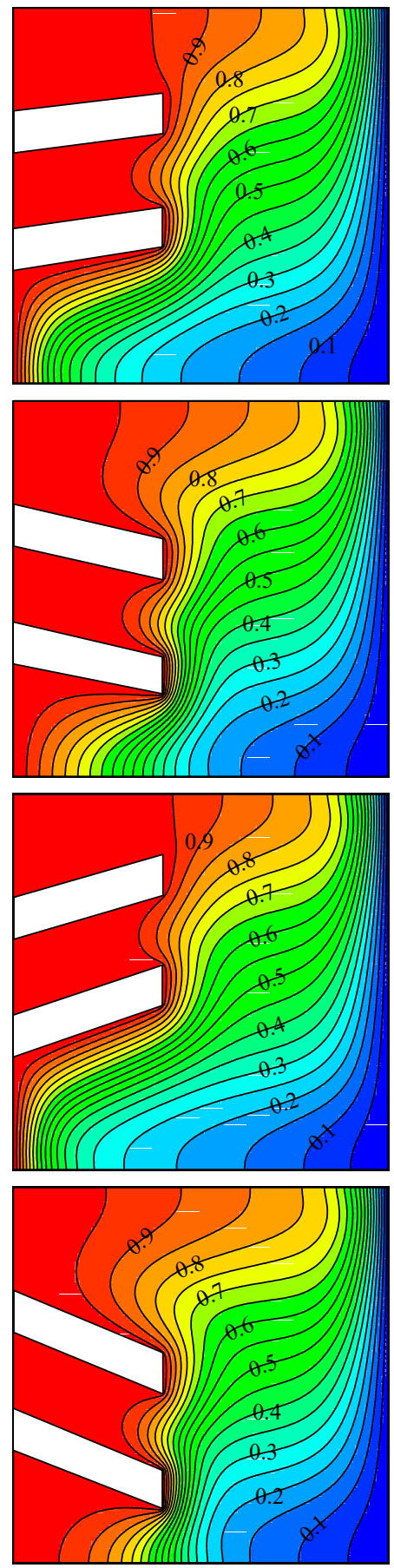

Figure 6. Cont. (c) $S_{G, T}$
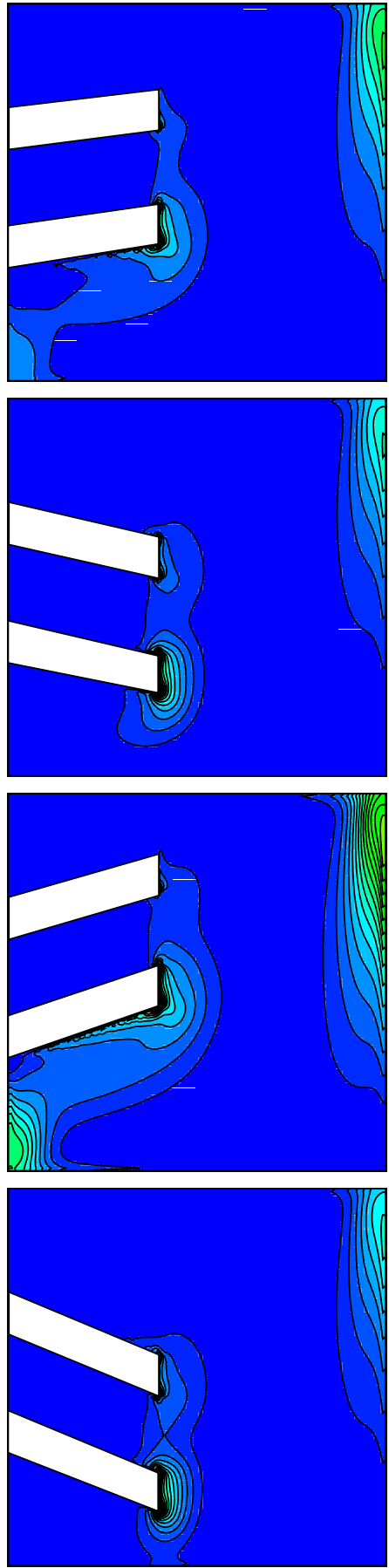
30
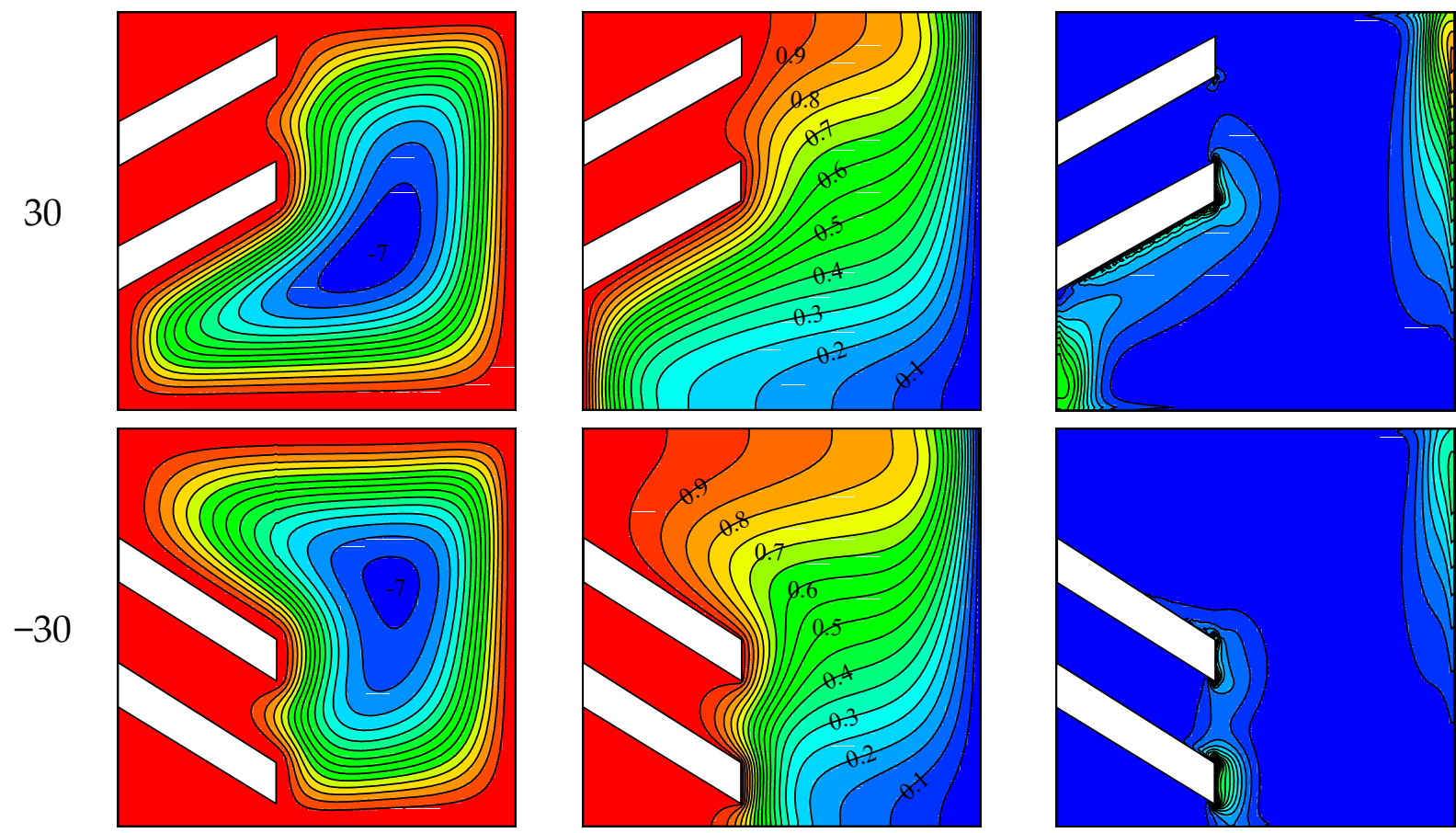

Figure 6. The (a) flow, (b) temperature and (c) entropy fields at different fin inclinations for $R a=10^{5}, H a=20, \varphi=0.03, \gamma=0^{\circ}$.

The variations of the dimensionless velocity (a) and dimensionless temperature (b) over the line $X=0.5$ are plotted in Figure 7 at different fin inclinations for the specified constants. In the dimensionless velocity diagram, tilting the fin toward the top and bottom of the cavity causes an increase in the maximum and minimum dimensionless velocities. This can be attributed to the increased distance of the fins from the center of the cavity, as well as the increased vortex velocity. Nonetheless, it must be noted that the orientation of the fins also plays an important role in the loci of maximum and minimum velocities $n$ the cavity. When the fin is tilted up, the maximum velocity occurs at the bottom of the cavity, while the highest velocity is found at the top when the fins are tilted down. According to the dimensionless temperature diagram, fluid temperature increases toward the top of the cavity due to the upward convection of hot fluid by buoyancy. Temperature changes with cavity inclination as a result of changes in the vortex velocity and fin location in the cavity.

The variations of (a) the local $\mathrm{Nu}$ over the cold wall of the cavity for negative and (b) the local $\mathrm{Nu}$ over the cold wall of the cavity for positive trigonometric fin inclination angles for $\mathrm{Ra}=10^{5}, \mathrm{Ha}=20, \varphi=0.03, \gamma=0^{\circ}$ are plotted in Figure 8 for the specified conditions. Figure 8 a shows that the most significant amount of $N u$ occurred at the top of the wall. In that area, the fluid inside the vortex, which is heated by the fins, strikes the cold wall for the first time, resulting in the highest HTR. Increasing the fin angle has slightly increased the $N u$. Figure $8 \mathrm{~b}$ also shows that the largest amount of $N u$ occurs in the upper area of the wall. Furthermore, increasing the angle in the negative direction of the fin increases the amount of heat transfer rate similar to that seen above. In the lower part of the wall, where the fluid has lost its temperature by moving along the side of the wall, the amount of heat transfer is less, and as a result, the lowest $N u$ is seen in that area.

The average $N u$ across the cold wall and GOE at different fin inclinations are plotted in Figure 9 for $\mathrm{Ra}=10^{5}, \mathrm{Ha}=20, \varphi=0.03, \gamma=0^{\circ}$. Increasing the fin inclination either in upward or downward directions enhances HTR and GOE. The vortex rotates faster with increases the distance of the tip of the fin from the center of the cavity. Furthermore, the faster vortex rotation causes an increase in the temperature difference, which, in turn, enhances the HTR. Considering the fact that GOE depends on the temperature and velocity gradients, GOE is also increased. 


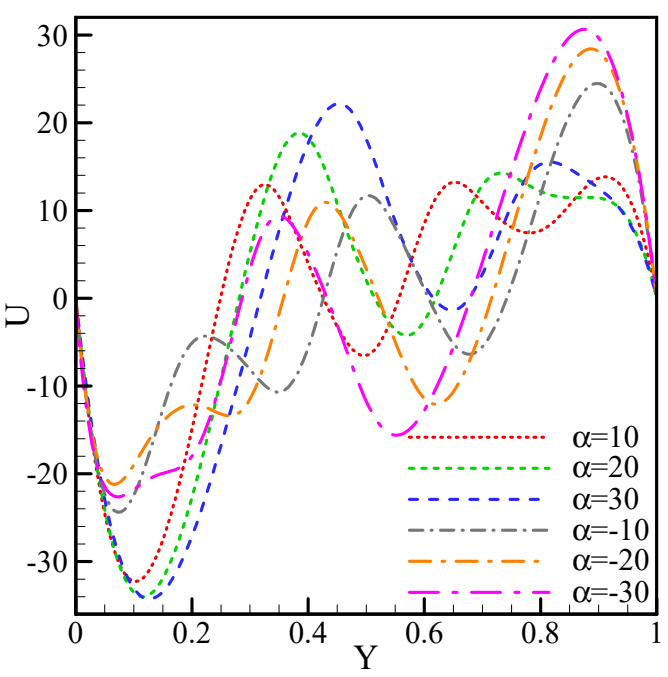

(a)

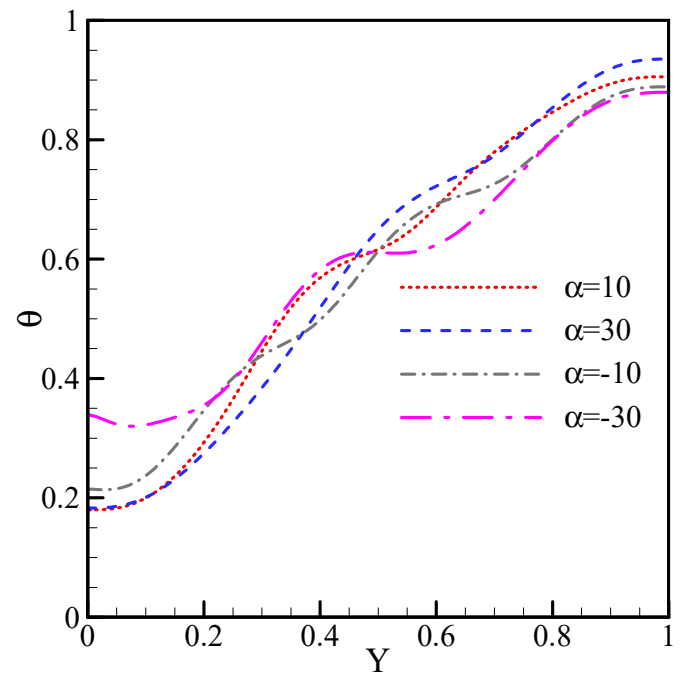

(b)

Figure 7. The variations of (a) the dimensionless velocity and (b) dimensionless temperature over the line $X=0.5$ at different fin inclinations for $\mathrm{R} a=10^{5}, \mathrm{Ha}=20, \varphi=0.03, \gamma=0^{\circ}$.

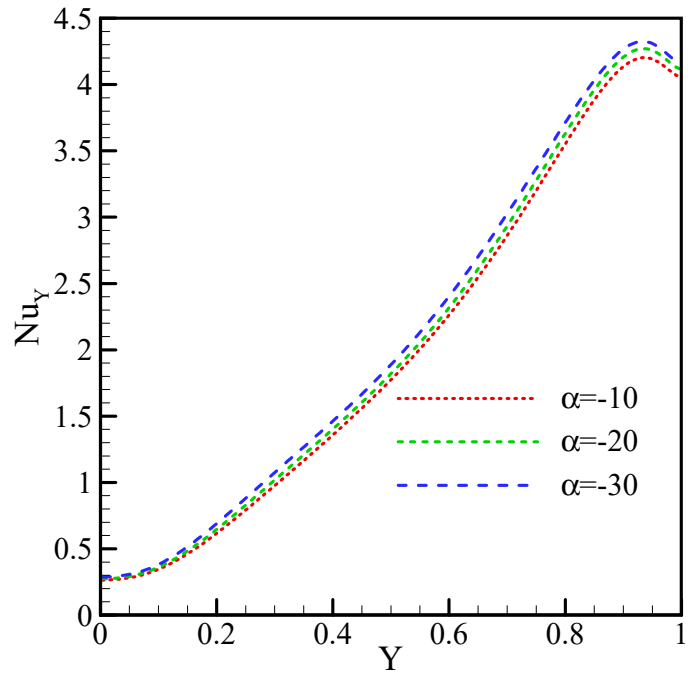

(a)

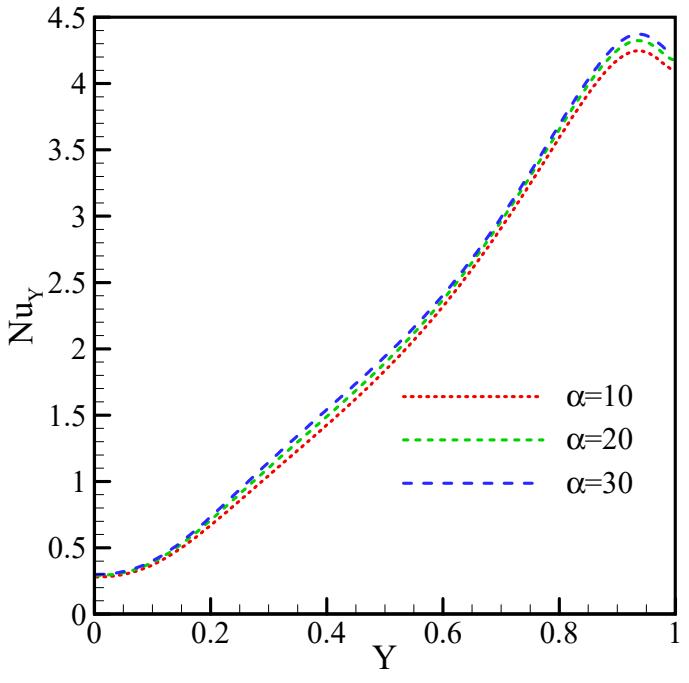

(b)

Figure 8. Variations of (a) the local $N u$ over the cold wall of the cavity for negative and (b) the local $N u$ over the cold wall of the cavity for positive trigonometric fin inclination angles at different fin inclinations for $R a=10^{5}, H a=20, \varphi=0.03, \gamma=0^{\circ}$.

\subsection{Effect of Fin Curvature}

The (a) flow, (b) temperature and (c) entropy fields are depicted in Figure 10 for the specified constants. Figure 10a shows that increasing the curvature of the fin increases the strength of the flow field. As mentioned, increasing the curvature of the fin increases the value of the maximum stream function in the middle of the vortex due to the increase in the distance between the tip of the fins and the middle of the cavity. Increasing the space for fluid movement has increased the speed of fluid movement. Thus, as can be seen in the streamlines contour, the speed of the vortex has increased with increasing fin curvature. Figure $10 \mathrm{~b}$ also shows slight changes in the isotherms with increasing fin curvature. These changes can be seen as a slight increase in the density of the lines in the areas under the fins. In Figure 10c, which is related to the entropy field, the compactness of the entropy production lines has increased with increasing curvature, which could indicate 
more entropy production, especially in those areas. Therefore, increasing the fin curvature can increase local entropy production.

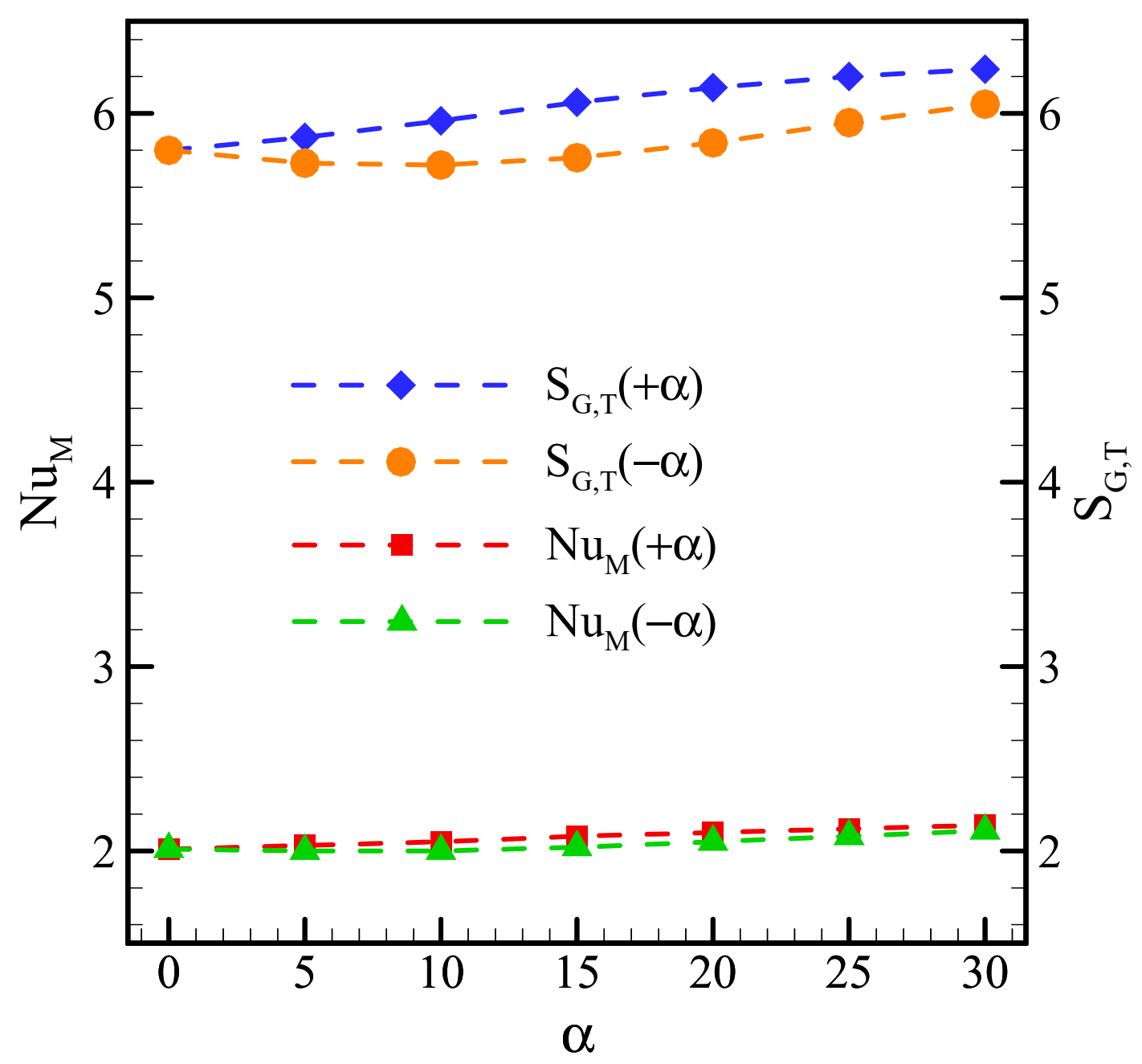

Figure 9. The average $N u$ over the cold wall and GOE at various fin inclinations for $R a=10^{5}, H a=20, \varphi=0.03, \gamma=0^{\circ}$.

The variations of the dimensionless velocity (a) and dimensionless temperature (b) over the line $X=0.5$ are plotted in Figure 11 for different fin curvatures and the specified constants. As seen, more significant maxima and minima dimensionless horizontal velocities are obtained with increasing fin curvatures. According to the flow field, this can be related to the higher vortex velocity due to an increase in the velocity in the cavity by increasing the curvature of the fins. Furthermore, with increasing the fin curvature, as their tips approach the upper parts of the cavity, velocity contours draw away from the left-side wall. The locus of maximum velocity is displaced toward the middle of the cavity. As seen in the dimensionless temperature diagram, the temperature decreases in the middle of the cavity but increases at the top by increasing the fin curvature. The tips of fins pulling away from the middle of the cavity and the displacement of the end parts of fins toward the top of the cavity are the main reasons behind these temperature variations.

The local $\mathrm{Nu}$ is plotted in Figure 12 for the specified constants. Apparently, the local heat transfer first increases at low curvatures, and the Nusselt number $(\mathrm{Nu})$ changes slightly at higher curvatures. The increased $N u$ can be attributed to the higher fluid velocity, particularly at lower fin curvatures. The final reduction of the local $\mathrm{Nu}$ at the top of the wall is the result of the vortex failing to reach the sharp corners of the cavity, which reduces fluid-wall collisions and, consequently, HTR. Nevertheless, the high-temperature gradient in this area still allows for a considerable HTR at the upper corner of the cavity. 
$\beta$

0.8

1.12

1.44
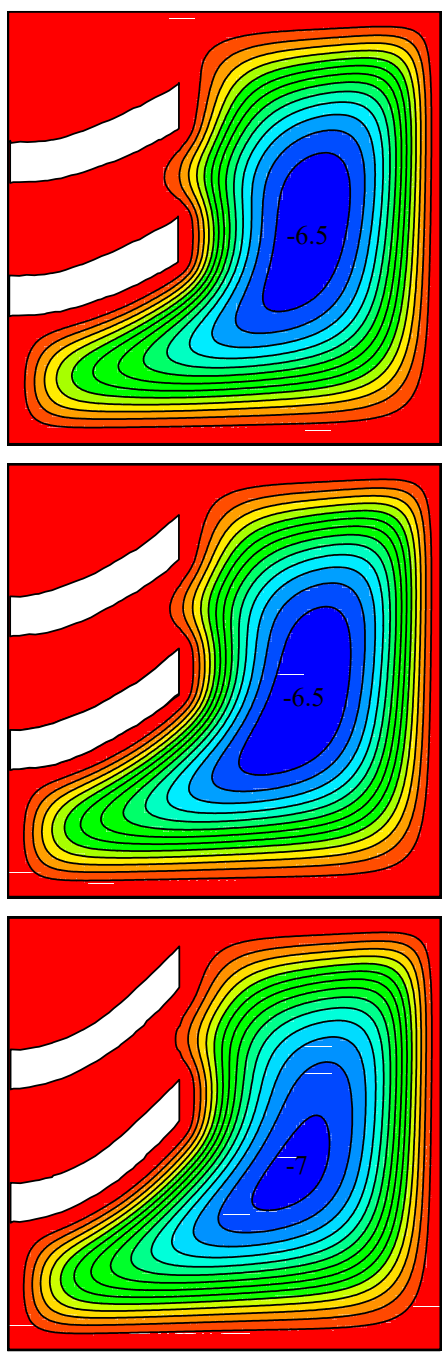

(b) $\mathrm{T}$
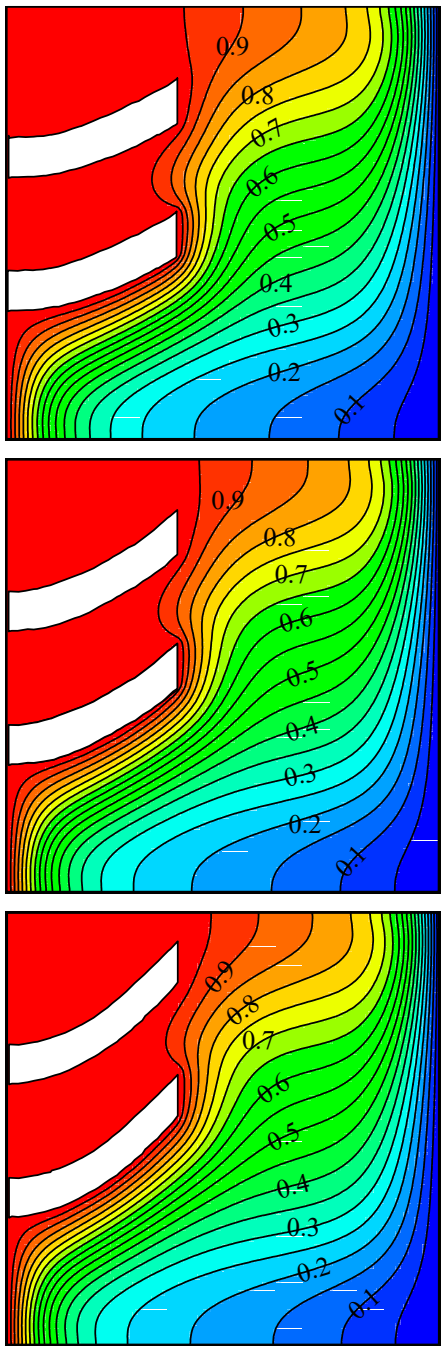

(c) $S_{G, T}$
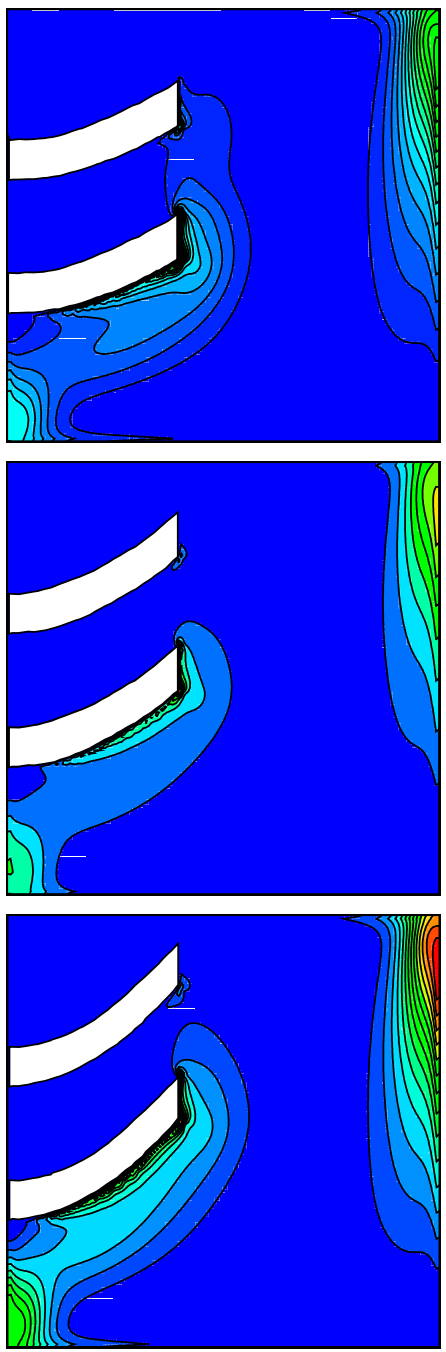

Figure 10. The (a) flow, (b) temperature and (c) entropy fields at different fin inclinations for $\mathrm{Ra}=10^{5}, \mathrm{Ha}=20, \varphi=$ $0.03, \gamma=0^{\circ}$.

The average $\mathrm{Nu}$ and GOE are presented in Table 2. Increasing the curvature, at first, enhances HTR and GOE. However, at $\beta=0.48$, increasing the curvature of the two fins causes a decrease in both parameters. A further increase in the fin curvature causes an increase in GOE and HTR. HTR and GOE strongly depend on the vortex velocity. Moving the ends of the fins away from the middle of the cavity accelerates the vortex. On the other hand, the limited reach of the flow toward the walls, particularly their middle parts, may reduce fluid-wall collisions and, consequently, the HTR. However, the changes in $N u$ and GOE with the fin curvature were found to be insignificant in the studied range (4.4 and 5.3 , respectively).

Table 2. The average $N u$ and GOE for different fin curvatures and $R a=10^{5}, H a=20, \varphi=0.03, \gamma=0^{\circ}$.

\begin{tabular}{cccccc}
\hline $\boldsymbol{\beta}$ & $\mathbf{0 . 1 6}$ & $\mathbf{0 . 4 8}$ & $\mathbf{0 . 8}$ & $\mathbf{1 . 1 2}$ & $\mathbf{1 . 4 4}$ \\
\hline$N u_{M}$ & 2.02 & 2.11 & 2.08 & 2.10 & 2.11 \\
$S_{G, T}$ & 5.84 & 6.15 & 6.04 & 6.11 & 6.14 \\
\hline
\end{tabular}




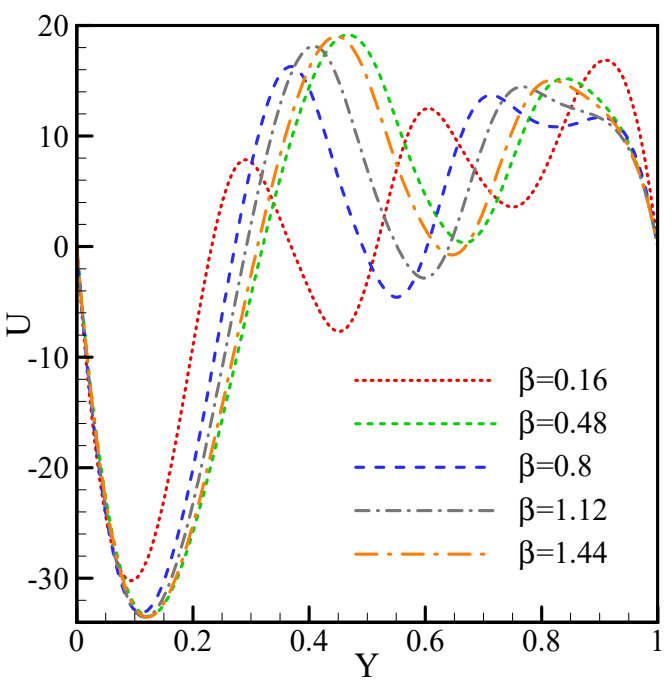

(a)

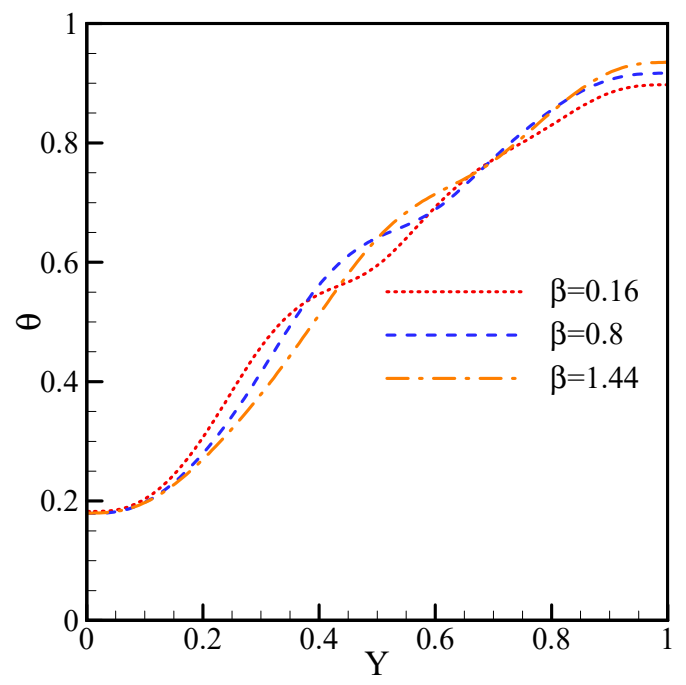

(b)

Figure 11. The variations of the (a) dimensionless velocity and (b) dimensionless temperature over the line $X=0.5$ at different fin curvatures and $\mathrm{Ra}=10^{5}, \mathrm{Ha}=20, \varphi=0.03, \gamma=0^{\circ}$.

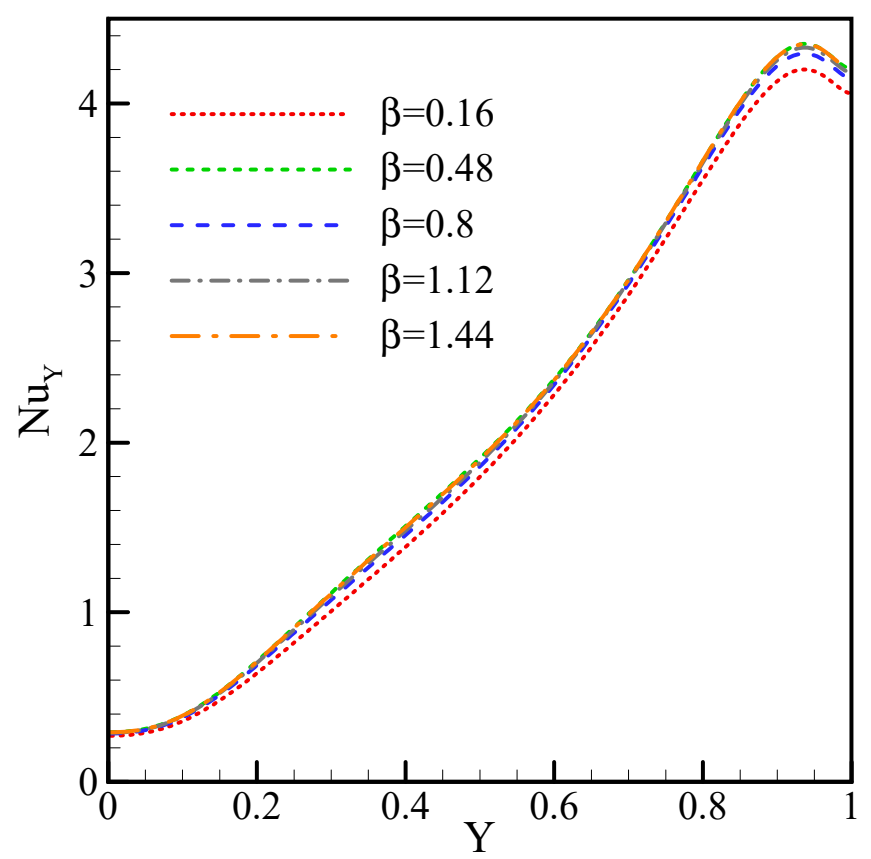

Figure 12. The local $N u$ over the cold wall for different fin curvatures and $R a=10^{5}, H a=20, \varphi=0.03$, $\gamma=0^{\circ}$.

\subsection{Effect of Cavity Inclination}

The flow field is depicted in Figure 13 at different cavity inclinations for $R a=10^{5}$, $H a=20, \varphi=0.03$ with (a) straight fins, (b) angular fins and (c) curved fins. Figure 13a shows that the angle of the cavity causes a large change in the strength of the vortex as well as the number of vortices. Comparing Figure $13 a-c$, it can be seen that the maximum stream function for angular and curved fins has greater values than the straight fin for any different angles of the cavity. In particular, the angular fin has a higher stream function value than the straight fin. It can also be seen that in the angular fin, the vortex has more penetration in the lower part of the fins and gets closer to the side wall. Additionally, for cases of curved and angular fins, less flow penetrates above the fin, so in this area, less fluid 
hits the hot wall with the upper wall of the fins compared to the straight fin. Changing the cavity angle can have a significant effect on the strength of the vortex because the position of the hot and cold walls changes relative to the gravity vector. In the horizontal cavity, the vortex strength is low, which increases with increasing angle. As when the cavity is horizontal (zero angle), the buoyancy force is less, so the amount of fluid displacement is less.

(a) Straight Fins
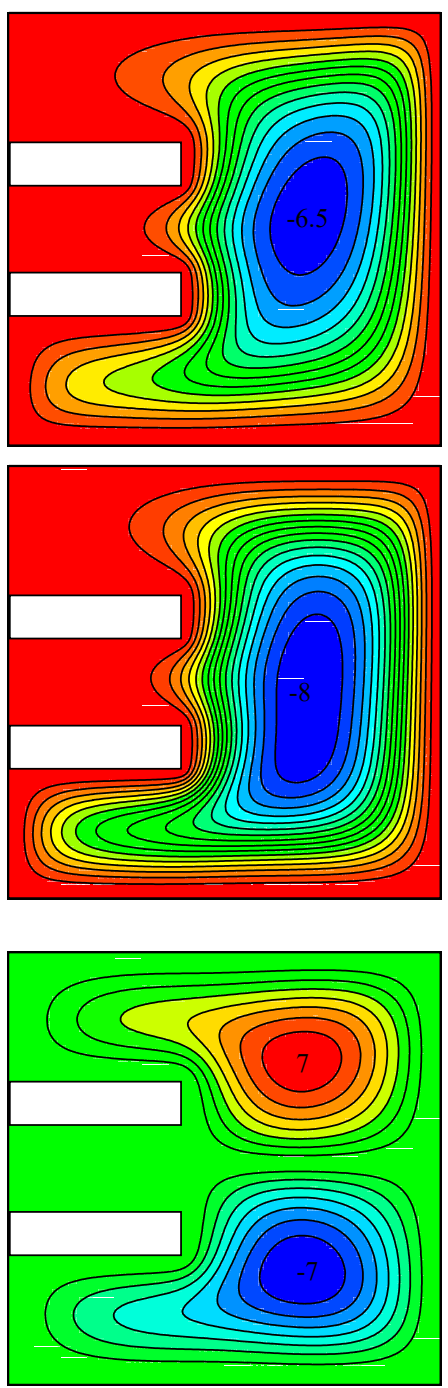

(b) Angular Fins
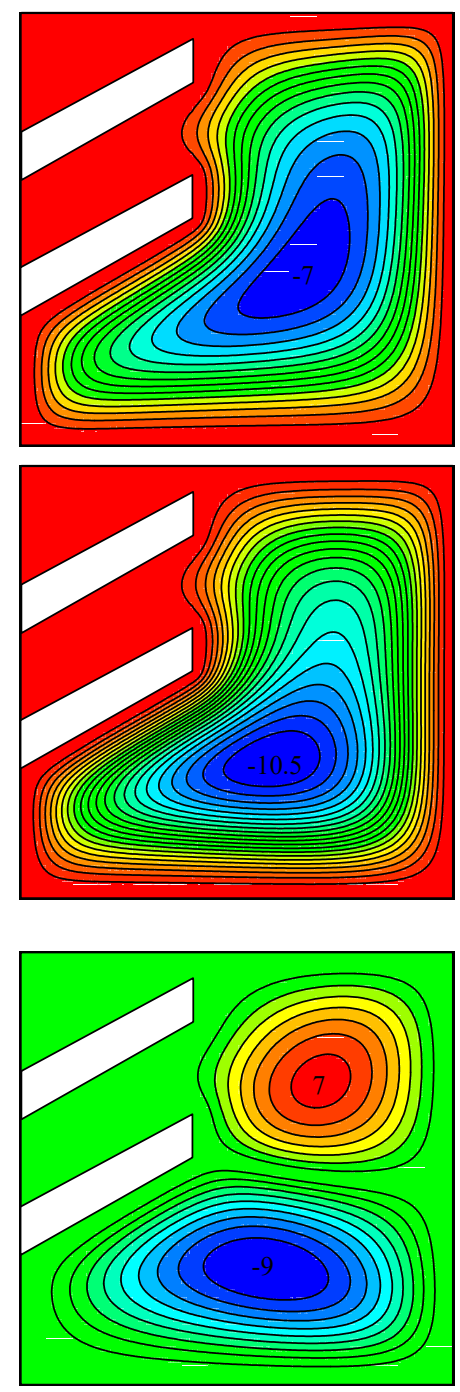

(c) Curve Fins
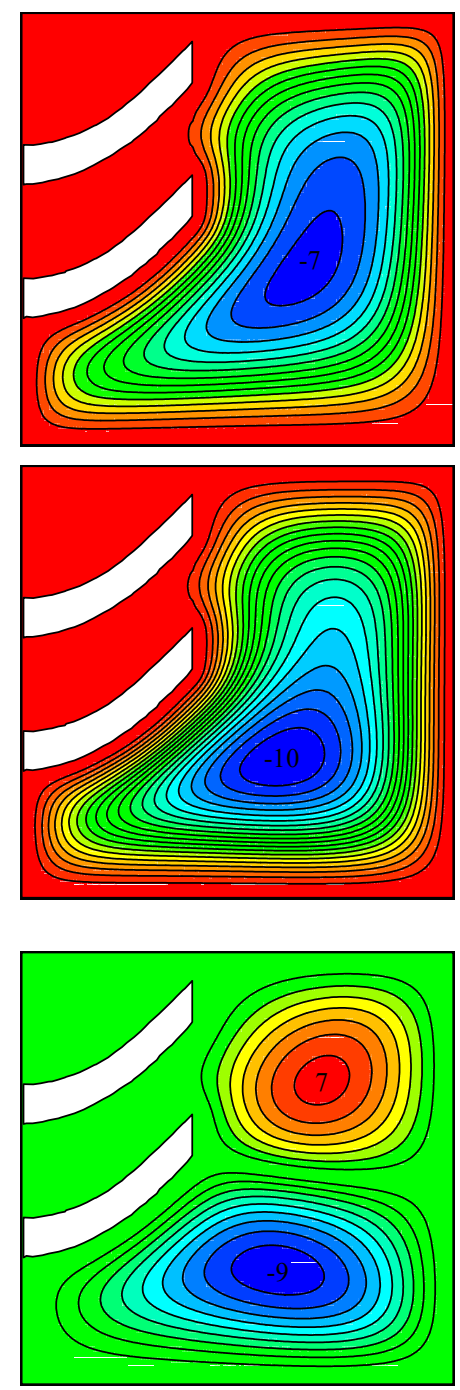

Figure 13. The flow field at different cavity inclinations for $R a=10^{5}, H a=20, \varphi=0.03$.

The temperature field is depicted in Figure 14 at different cavity inclinations for $R a=10^{5}, H a=20, \varphi=0.03$ with (a) straight fins, (b) angular fins and (c) curved fins Comparing Figure $14 \mathrm{a}, \mathrm{c}$, it can be seen that for the horizontal cavity in cases of angular and curved fins, the density of isotherms is higher than that of the straight fin at the bottom of the fins. In this area, due to higher penetration, the amount of temperature change in the angular and curved fins is more than the straight fin. However, in these two fins, the flow penetration in the middle of the fins is less than the straight fin, and fewer isotherms are seen in this area. In the vertical cavity, the penetration of isotherms between the angular and curved fins is greater than in the straight case. In fact, for cases of angular and curved fins, the fluid moves from the top of the first fin to the cold wall, while for the case of straight fins, the fluid moves through the two fins to the cold wall. By changing the angle of the cavity, the place of density of the isotherms also changes and their curvature is in 
the direction of fluid motion. In the horizontal cavity, the fluid moves along the walls, resulting in greater curvature of the isotherms near the walls. In the vertical cavity, in all three cases, the fluid rises from the middle of the cavity and descends from its sides. Hence, the curvature of isotherms in the middle of the cavity is greater than that in other areas. Additionally, in the vertical cavity, the highest density of isotherms is in the middle of the cold wall, while in other cases, this density was seen on the upper part of the cold wall. As in the vertical cavity (at an angle of 90), the temperature gradient is high in the middle of the wall, but in other cases it is higher in the upper areas of the wall. In the vertical cavity, isotherms are also seen in the direction of straight fins, but in the other two cases, isotherms are seen asymmetrically due to the inclination of the fins to one side.

(a) Straight Fins
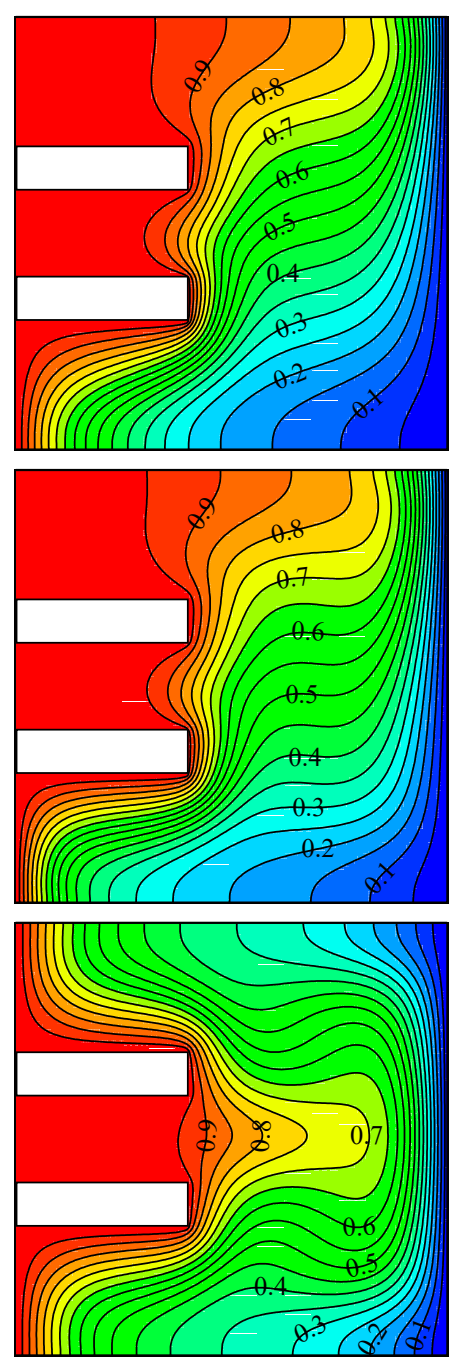

(b) Angular Fins
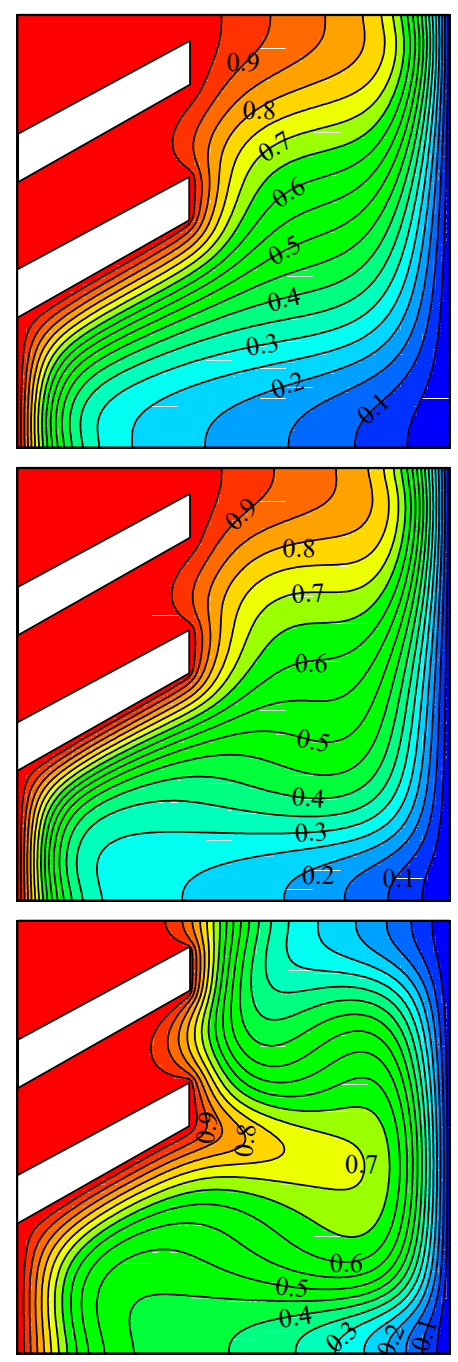

(c) Curved Fins
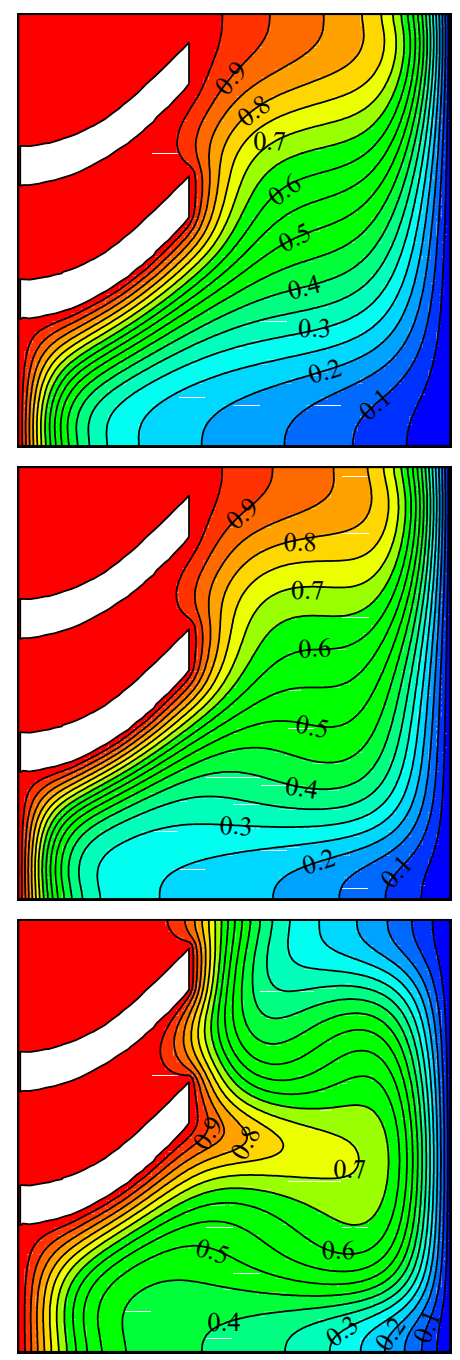

Figure 14. The temperature field at different cavity inclinations for $R a=10^{5}, H a=20, \varphi=0.03$.

The entropy field is depicted in Figure 15 at different cavity inclinations for $R a=10^{5}$, $H a=20, \varphi=0.03$ with (a) straight fins, (b) angular fins and (c) curved fins. Comparing Figure $15 \mathrm{a}$ to $15 \mathrm{c}$ it is observed that the highest local entropy production occurs at the corners of the straight fin, while in the angular fin, the highest local entropy production is observed on the lower wall of the lower fin. In Figure 15c, which is related to the curved fin, the highest entropy production is observed on the lower wall and the vertical wall of the lower fin. In these places, due to the high temperature gradient, the amount of entropy production is also high. In the vertical cavity, the maximum local entropy production 
(a) Straight Fins
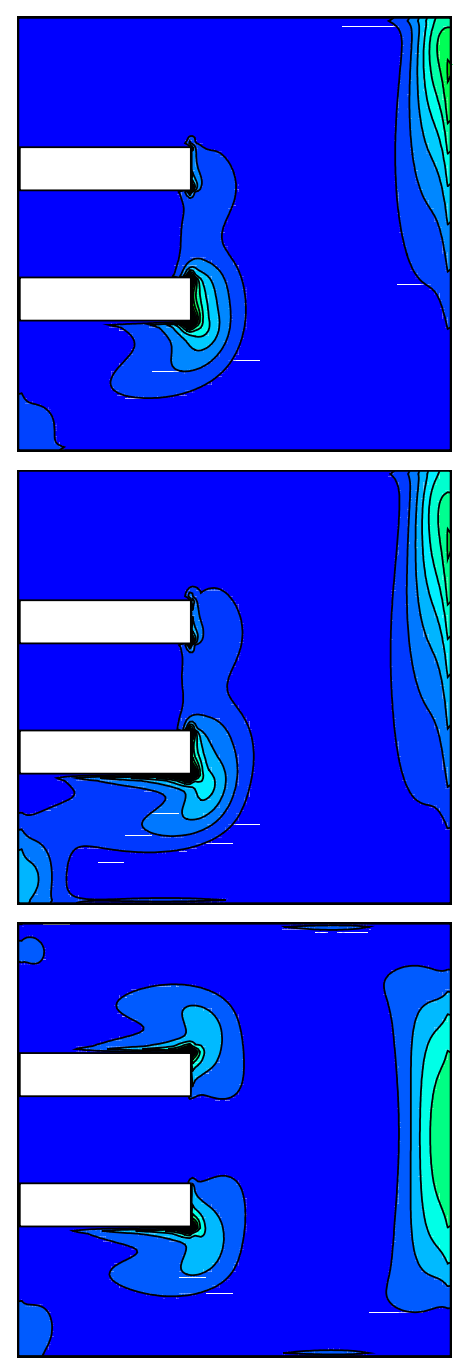

(b) Angular Fins
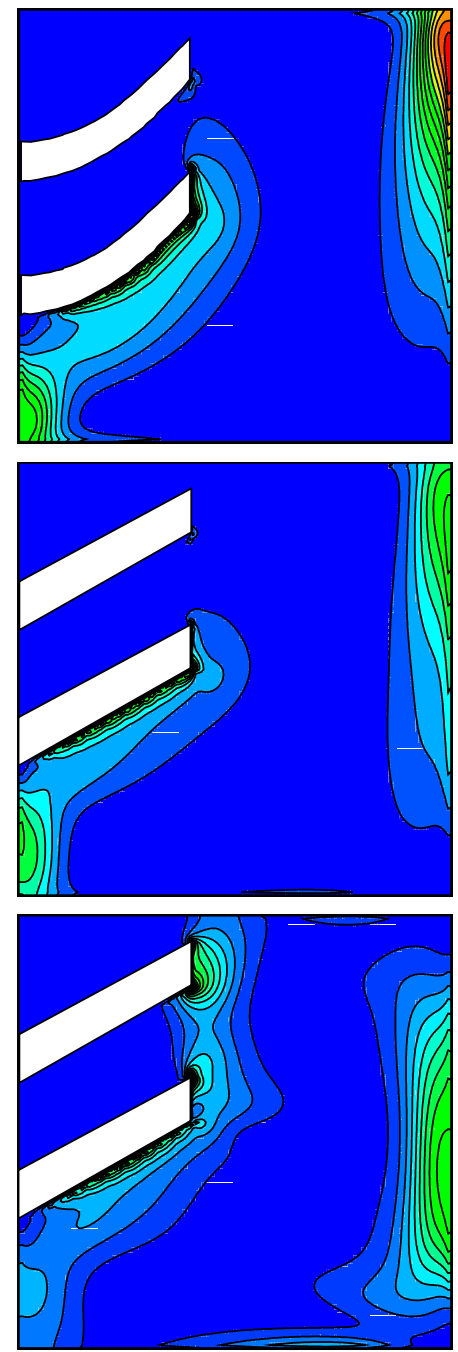

(c) Curved Fins
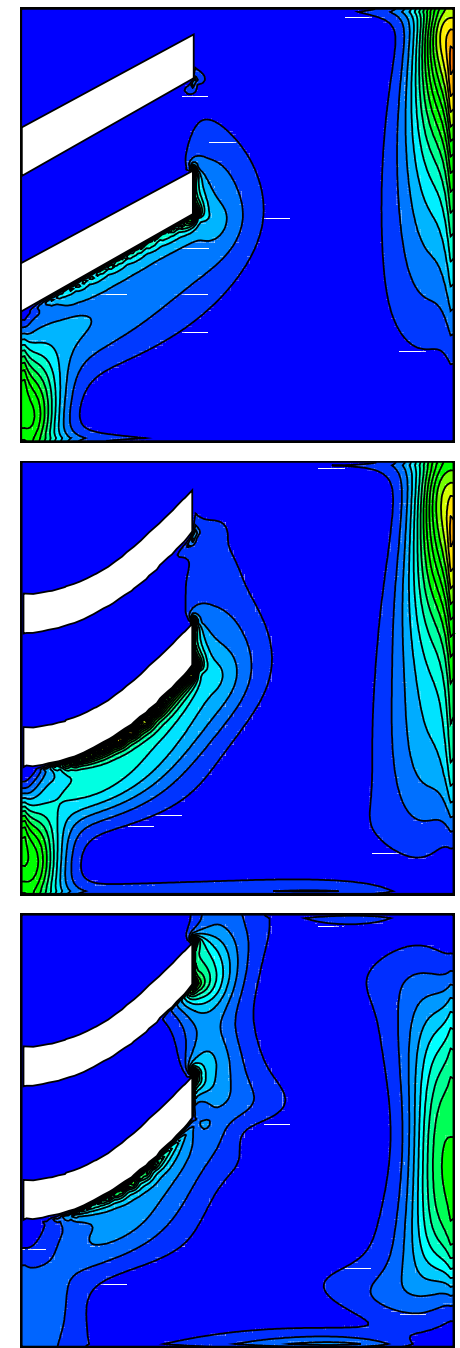

Figure 15. The entropy field at different cavity inclinations for $\mathrm{R} a=10^{5}, \mathrm{Ha}=20, \varphi=0.03$.

The dimensionless horizontal velocity is plotted in Figure 16 over the line $X=0.5$ for different cavity inclinations and $\mathrm{Ra}=10^{5}, \mathrm{Ha}=20, \varphi=0.03$ with (a) straight fins (b) inclined fins, and (c) curved fins. The velocity plots corresponding to the curved and inclined fins are similar but differ from those for the straight fins. In all three cases, higher velocity maxima and minima were achieved at a cavity angle of $90^{\circ}$. The velocity increases at higher inclinations-particularly in the middle of the cavity. For the straight fin, the velocity minima near the walls are also higher. Meanwhile, for the other two fin configurations, the flow direction changes near the top of the cavity. As a result, near the top of the vertical cavity, the velocity is minimum instead of maximum, suggesting the reversal of the direction of velocity. 


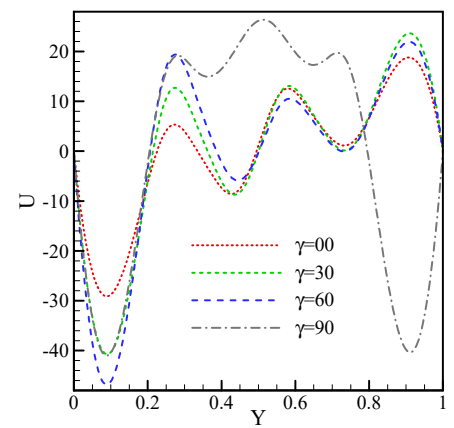

(a)

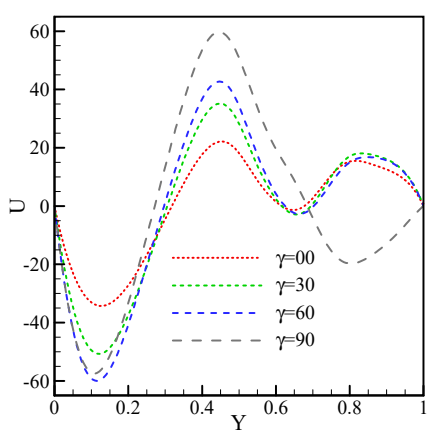

(b)

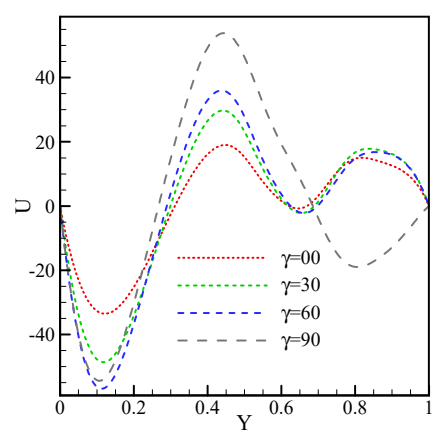

(c)

Figure 16. The dimensionless horizontal velocity over the line $X=0.5$ for different cavity inclinations and $R a=10^{5}, H a=20$, $\varphi=0.03$ with (a) straight fins, (b) inclined fins and (c) curved fins.

The dimensionless temperature is plotted in Figure 17 for the specified conditions. The dimensionless temperature plots also show similar trends for the curved and inclined fins that are different from those for the straight fin. Similarly, the temperature rises considerably in the middle of the vertical cavity. The hot fluid flow through the middle of the cavity instead of the sides causes an increase in the temperature in the middle and a significant reduction in the temperature near the upper parts since the cold fluid moves toward the hot wall near the top of the vertical cavity. Increasing the inclination of the cavity from $0^{\circ}$ increases the temperature in its lower parts, indicating a higher fluid temperature near the bottom wall.

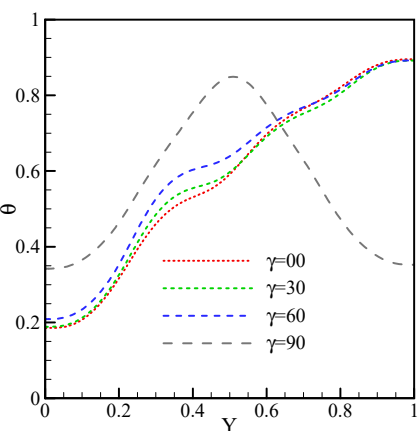

(a)

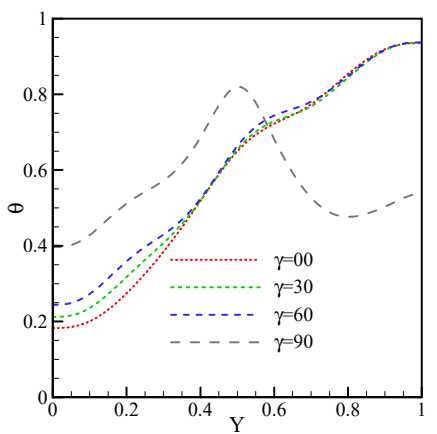

(b)

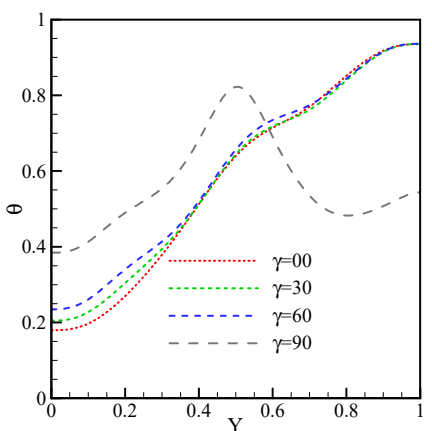

(c)

Figure 17. The dimensionless temperature over the line $X=0.5$ for different cavity inclinations and $\mathrm{R} a=10^{5}, \mathrm{Ha}=20, \varphi=0.03$ with (a) straight fins, (b) inclined fins and (c) curved fins.

The local $\mathrm{Nu}$ is plotted in Figure 18 for the specified conditions. All local $\mathrm{Nu}$ plots are similar, showing smaller values at the bottom of the wall and higher ones on top for all cavity inclinations, except for $90^{\circ}$, due to the considerable or small temperature difference in that area. Contrary to the other cases, the $N u$ is higher in the middle of the wall and lower at its beginning or end of the vertical cavity. The temperature difference and, consequently, HTR are enhanced in this case due to the hot fluid coming in contact with the middle parts of the wall. Increasing the inclination, overall, improved HTR at the bottom of the wall and reduced it on top. These changes can be attributed to temperature difference variations in different cases that affect the local $N u$.

GOE and HTR are plotted in Figure 19 for the specified conditions. As clearly seen, the maximum HTR is achieved in the inclined cavity using curved and inclined fins, while HTR is maximized with straight fins in the vertical cavity. Apparently, the best configuration for heat transfer through curved fins is one with a curved cavity. According to the entropy diagram, the inclined cavity offers the highest GOE in all cases, and the maximum GOE is achieved with curved fins. GOE is drastically reduced at a cavity angle of $90^{\circ}$ as a result 
of splitting of the vortex into two vortices. This, in turn, affects the velocity gradient, limiting GOE.

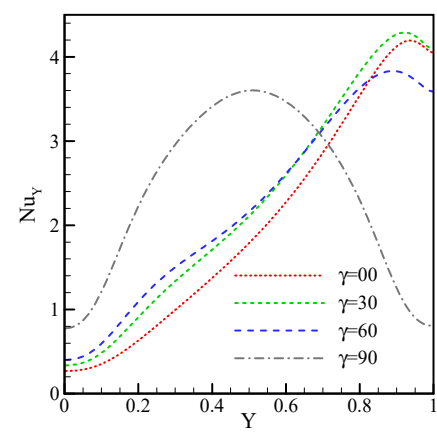

(a)

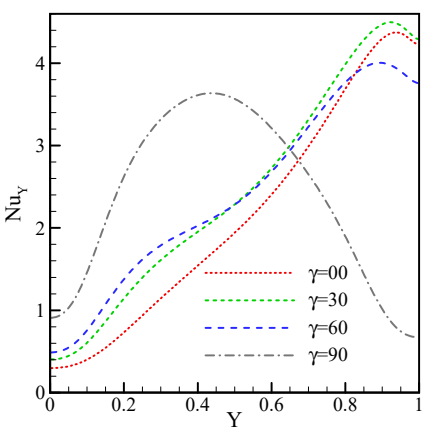

(b)

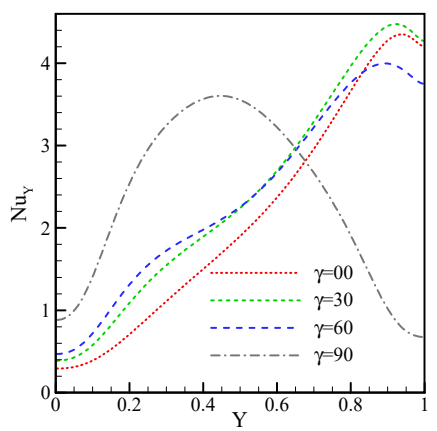

(c)

Figure 18. The local $N u$ over the right-side wall for different cavity inclinations and $R a=10^{5}, H a=20, \varphi=0.03$ with (a) straight fins, (b) inclined fins and (c) curved fins.

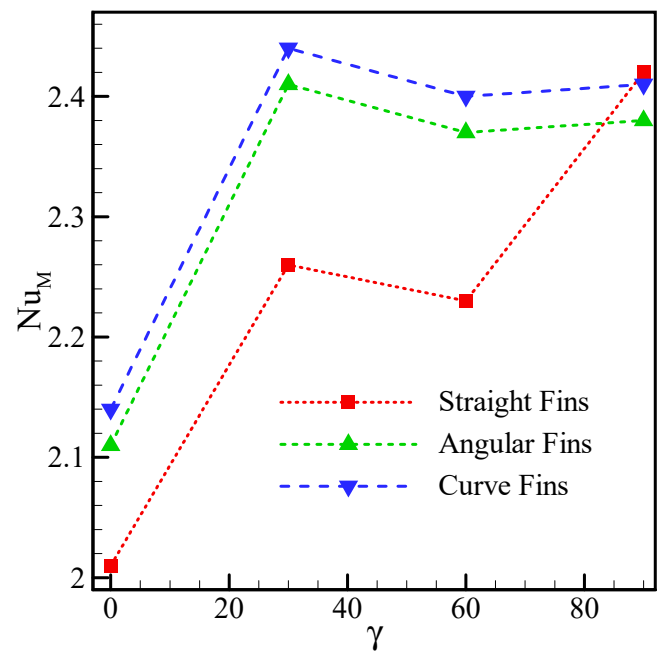

(a)

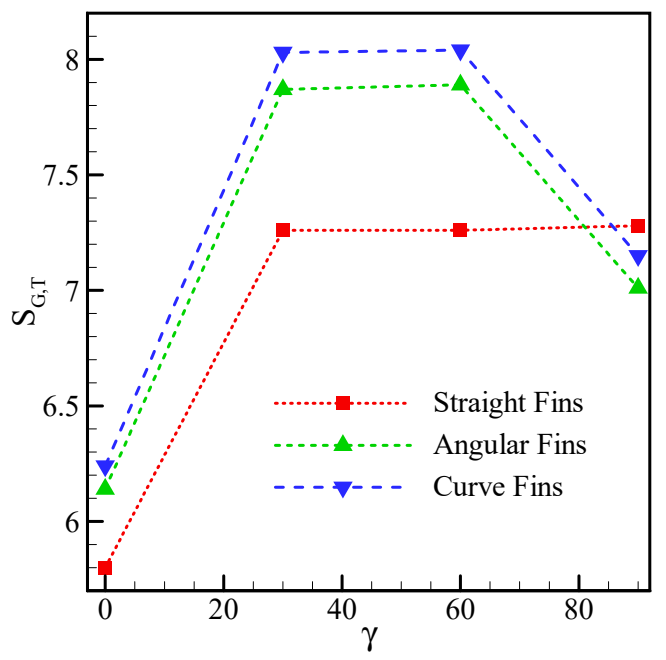

(b)

Figure 19. The (a) average $N u$ and (b) GOE at different cavity inclinations for $R a=10^{5}, H a=20, \varphi=0.03$ and the three fin configurations.

Table 3 presents the maximum and minimum HTR and entropy, showing the highest heat transfer (2.46) was achieved with the curved fin at a cavity inclination of $45^{\circ}$ and the least GOE (5.8) with the straight fin at $0^{\circ}$.

Table 3. The maximum and minimum HTR and GOE for the three fin configurations and $R a=10^{5}$, $H a=20, \varphi=0.03$.

\begin{tabular}{ccccc}
\hline & $\mathbf{M a x} \mathbf{N u}_{\mathbf{M}}$ & $\mathbf{M i n} \mathbf{N u}_{\mathbf{M}}$ & $\mathbf{M a x} \mathbf{S}_{\mathbf{G}, \mathbf{T}}$ & $\mathbf{M i n} \mathbf{S}_{\mathbf{G}, \mathbf{T}}$ \\
\hline Straight Fins & $2.42(\gamma=90)$ & $2.01(\gamma=00)$ & $7.28(\gamma=90)$ & $5.80(\gamma=00)$ \\
\hline Angular Fins & $2.43(\gamma=45)$ & $2.14(\gamma=00)$ & $8.12(\gamma=45)$ & $6.24(\gamma=45)$ \\
\hline Curve Fins & $2.46(\gamma=45)$ & $2.11(\gamma=00)$ & $8.04(\gamma=60)$ & $6.14(\gamma=60)$ \\
\hline
\end{tabular}




\subsection{Effect of $\mathrm{Ha}$}

Figure 20 shows the (a) dimensional vertical velocity and (b) dimensional temperature at $Y=0.5$ for $R a=10^{5}, \gamma=0, \varphi=0.03$ and straight fins for different $\mathrm{Ha}$. Figure 20a shows that increasing the Hartmann number reduces the velocity values in the cavity. Increasing the Hartmann number increases the Lorentz force and thus decreases the strength of the vortices. Hence, the amount of velocity is reduced. As the velocity decreases, it can be seen that the amount of the velocity is tending to zero. Figure 20b also shows that increasing the Hartmann number reduces the curvature of the temperature graph due to the smaller vortex, which causes the temperature changes in the cavity to be less than before. In this case, the temperature graph is becoming linear. While the temperature lines are linear, the conduction heat transfer in the cavity is predominant.

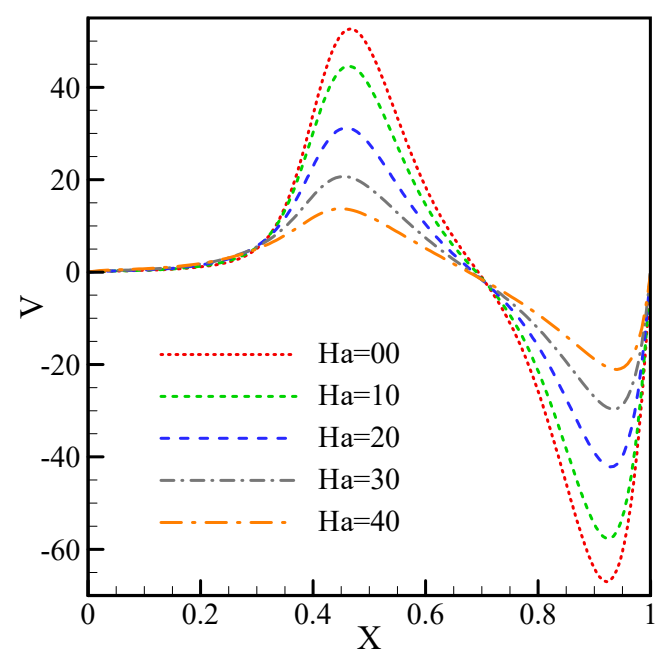

(a)

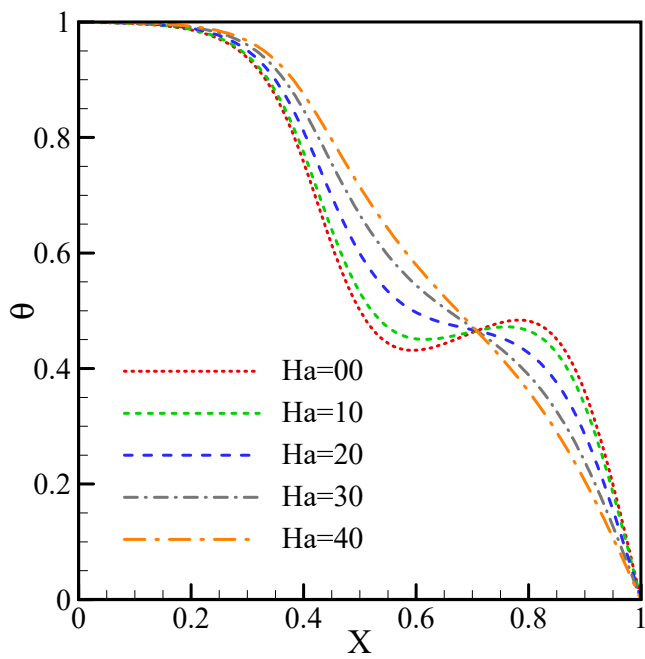

(b)

Figure 20. The (a) dimensionless velocity and (b) dimensional temperature at $Y=0.5$ for $R a=10^{5}, \gamma=0, \varphi=0.03$ and straight fins for different $\mathrm{Ha}$.

Table 4 reports the effect of the magnetic field on the GOE and the $N u$ for the straight fin case at different Hartmann numbers and the volume fraction of nanoparticles of $3 \%$ for the horizontal cavity. It can be seen that increasing the Hartmann number has reduced both the values of the $\mathrm{Nu}$ and GOE, so that reaching the Hartmann number to 40 has reduced the value of the $\mathrm{Nu}$ by $37.9 \%$ and the GOE by $33.8 \%$. The reduction in vortex velocity has been the main reason for this decrease because it has reduced both the amounts of HTR and GOE by velocity changes.

Table 4. Effect of the magnetic field on the GOE and the $N u$ for the straight fin case at different Hartmann numbers and the volume fraction of nanoparticles of $3 \%$ for the horizontal cavity.

\begin{tabular}{cccccc}
\hline $\mathbf{H a}$ & $\mathbf{0}$ & $\mathbf{1 0}$ & $\mathbf{2 0}$ & $\mathbf{3 0}$ & $\mathbf{4 0}$ \\
\hline$N u_{M}$ & 2.37 & 2.26 & 2.01 & 1.72 & 1.47 \\
\hline$\%$ change $N u_{M}$ & 0.0 & 4.6 & 15.1 & 27.4 & 37.9 \\
\hline$S_{G, T}$ & 6.50 & 6.33 & 5.80 & 5.07 & 4.30 \\
\hline$\%$ change $S_{G, T}$ & 0.0 & 2.6 & 10.7 & 22.0 & 33.8 \\
\hline
\end{tabular}

Finally, by comparing the present results in this work with the same work as in the past [25], it can be seen that by changing the conditions, the values of heat transfer in the cavity can be improved compared to the reference [25]. Alnaghi et al. [25] have studied a cavity equipped with two straight fins on a hot wall and have also considered the effects of a magnetic field. Compared to this work, it can be seen that by increasing the angle of the 
fin, the amount of heat transfer can be increased by $6.4 \%$ of the horizontal fin ratio. It is also possible to increase the amount of heat transfer by $4.9 \%$ compared to this article by bending the fins.

\section{Conclusions}

In this paper, the effect of installing three different types of fins on the wall of a square cavity on free convective heat transfer has been studied. These three types of fins include straight fins, inclined fins and curved fins. The cavity is affected by the magnetic field. By changing parameters such as fin angle, fin curvature, cavity angle and Hartmann number, the following results are obtained:

1. The maximum values of $N u$ and GOE are, respectively, 2.11 and $6.15 \mathrm{~W} / \mathrm{s}$, which occurs in the dimensionless curvature of the fin equal to 0.48 .

2. The highest and lowest HTRs belong to curved fins at a cavity angle of 45 and straight fins at an angle of zero, respectively.

3. As the fin angle increases, the HTR and GOE increase.

4. By examining the cavity angle, the highest entropy production was observed for angled fins at a cavity angle of 45 . The lowest rate occurs in straight fins at a cavity angle of zero.

5. In the horizontal cavity, by changing the fin angle, the heat transfer can be increased by a maximum of $6.4 \%$, while it can be increased by a maximum of $4.9 \%$ by bending the fins.

6. With increasing the Hartmann number from 0 to $40, N u$ and GOE decreased by $37.9 \%$ and $33.8 \%$, respectively.

Author Contributions: Conceptualization, Y.K.; Data curation, Y.K. and A.A.A.; Formal analysis, Y.K., H.A. and A.A.A.; Investigation, Y.K., A.A.A., H.A. and A.A.; Methodology, Y.K., A.A.A. and A.A.; Supervision, Y.K., M.S. and G.C.; Writing-original draft, Y.K., A.A.A. and A.A.; Writingreview \& editing, A.A., M.S. and G.C. All authors have read and agreed to the published version of the manuscript.

Funding: This research received no external funding.

Institutional Review Board Statement: Not applicable.

Informed Consent Statement: Not applicable.

Data Availability Statement: Not applicable.

Acknowledgments: This work was supported by the Taif University Researchers Supporting Project, Taif University, Taif, Saudi Arabia, under Project TURSP-2020/121.

Conflicts of Interest: The authors declare no conflict of interest.

\section{Nomenclature}

$B_{0} \quad$ Magnetic field strength

$C_{p} \quad$ Specific heat $[\mathrm{J} /(\mathrm{kg} \cdot \mathrm{K})]$

$g \quad$ Gravitational acceleration $\left[\mathrm{m} / \mathrm{s}^{2}\right]$

$h \quad$ Heat transfer coefficient $\left[\mathrm{w} /\left(\mathrm{m}^{2} \cdot \mathrm{K}\right)\right]$

$H \quad$ Enclosure non-dimensional length [-]

$\mathrm{Ha}$ Hartmann number $\left(\sqrt{\frac{\sigma_{f} B_{0}^{2} l^{2}}{\mu_{f}}}\right)$

$k \quad$ Thermal conductivity $[\mathrm{W} /(\mathrm{m} \cdot \mathrm{K})]$

$l \quad$ Enclosure length [m]

$\theta$

$\mathrm{Nu}$ Nusselt number [-]

$p \quad$ Pressure $[\mathrm{Pa}] \quad \rho$

$P \quad$ Non-dimensional pressure $\left(\bar{P} l^{2} / \rho_{\mathrm{nf}} \alpha_{\mathrm{f}}^{2}\right) \quad \sigma$

$\begin{array}{ll}\begin{array}{ll}\text { Greek symbols } \\ \alpha\end{array} & \text { Fin angle } \\ \beta & \text { Curve fin parameter } \\ \gamma & \text { Cavity angle }\left[^{\circ}\right] \\ \zeta & \text { Irreversibility } \\ \theta & \text { distribution ratio } \\ \mu & \text { Temperature } \\ \rho & \text { Dynamic viscosity } \\ \sigma & {[\mathrm{W} /(\mathrm{m} \cdot \mathrm{K})]} \\ & \text { Density }\left[\mathrm{Kg} / \mathrm{m}^{3}\right] \\ & \text { Electrical } \\ & \text { conductivity }[\Omega \cdot \mathrm{m}]\end{array}$




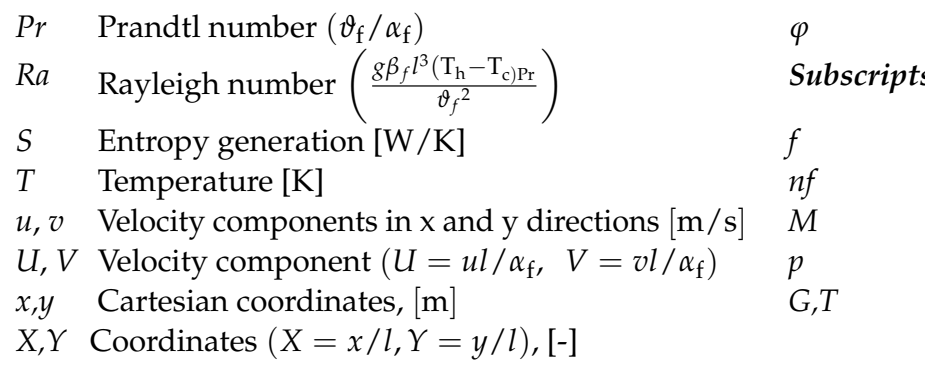

Solid volume fraction

Base fluid

Nanofluid

Average

Particle

Total generation

\section{References}

1. Parsa, S.M.; Javadi, D.; Rahbar, A.; Majidniya, M.; Salimi, M.; Amidpour, Y.; Amidpour, M. Experimental investigation at a summit above 13,000 ft on active solar still water purification powered by photovoltaic: A comparative study. Desalination 2020 476, 114146. [CrossRef]

2. Parsa, S.M.; Javadi, D.; Rahbar, A.; Majidniya, M.; Aberoumand, S.; Amidpour, Y.; Amidpour, M. Experimental assessment on passive solar distillation system on Mount Tochal at the height of $3964 \mathrm{~m}$ : Study at high altitude. Desalination 2019, 466, 77-88. [CrossRef]

3. Aghakhani, S.; Pordanjani, A.H.; Karimipour, A.; Abdollahi, A.; Afrand, M. Numerical investigation of heat transfer in a power-law non-Newtonian fluid in a C-Shaped cavity with magnetic field effect using finite difference lattice Boltzmann method. Comput. Fluids 2018, 176, 51-67. [CrossRef]

4. Pordanjani, A.H.; Aghakhani, S.; Afrand, M.; Mahmoudi, B.; Mahian, O.; Wongwises, S. An updated review on application of nanofluids in heat exchangers for saving energy. Energy Convers. Manag. 2019, 198, 111886. [CrossRef]

5. Salat, J.; Xin, S.; Joubert, P.; Sergent, A.; Penot, F.; Le Quéré, P. Experimental and numerical investigation of turbulent natural convection in a large air-filled cavity. Int. J. Heat Fluid Flow 2004, 25, 824-832. [CrossRef]

6. Parsa, S.M.; Rahbar, A.; Koleini, M.; Javadi, Y.D.; Afrand, M.; Rostami, S.; Amidpour, M. First approach on nanofluid-based solar still in high altitude for water desalination and solar water disinfection (SODIS). Desalination 2020, 491, 114592. [CrossRef]

7. Parsa, S.M.; Rahbar, A.; Koleini, M.; Aberoumand, S.; Afrand, M.; Amidpour, M. A renewable energy-driven thermoelectricutilized solar still with external condenser loaded by silver/nanofluid for simultaneously water disinfection and desalination. Desalination 2020, 480, 114354. [CrossRef]

8. Parsa, S.M.; Yazdani, A.; Dhahad, H.; Alawee, W.H.; Hesabi, S.; Norozpour, F.; Javadi, D.; Ali, H.M.; Afrand, M. Effect of Ag, Au, $\mathrm{TiO}_{2}$ metallic/metal oxide nanoparticles in double-slope solar stills via thermodynamic and environmental analysis. J. Clean. Prod. 2021, 311, 127689. [CrossRef]

9. Giwa, S.O.; Sharifpur, M.; Ahmadi, M.H.; Murshed, S.M.S.; Meyer, J.P. Experimental Investigation on Stability, Viscosity, and Electrical Conductivity of Water-Based Hybrid Nanofluid of MWCNT-Fe ${ }_{2} \mathrm{O}_{3}$. Nanomaterials 2021, 11, 136. [CrossRef]

10. Giwa, S.; Sharifpur, M.; Ahmadi, M.H.; Meyer, J.P. A review of magnetic field influence on natural convection heat transfer performance of nanofluids in square cavities. J. Therm. Anal. Calorim. 2020, 1-43. [CrossRef]

11. Mahdavi, M.; Sharifpur, M.; Ahmadi, M.H.; Meyer, J.P. Nanofluid flow and shear layers between two parallel plates: A simulation approach. Eng. Appl. Comput. Fluid Mech. 2020, 14, 1536-1545. [CrossRef]

12. Mahdavi, M.; Garbadeen, I.; Sharifpur, M.; Ahmadi, M.H.; Meyer, J.P. Study of particle migration and deposition in mixed convective pipe flow of nanofluids at different inclination angles. J. Therm. Anal. Calorim. 2019, 135, 1563-1575. [CrossRef]

13. Mahdavi, M.; Sharifpur, M.; Ahmadi, M.H.; Meyer, J.P. Aggregation study of Brownian nanoparticles in convective phenomena. J. Therm. Anal. Calorim. 2019, 135, 111-121. [CrossRef]

14. Jahanshahi, M.; Hosseinizadeh, S.; Alipanah, M.; Dehghani, A.; Vakilinejad, G. Numerical simulation of free convection based on experimental measured conductivity in a square cavity using Water $/ \mathrm{SiO}_{2}$ nanofluid. Int. Commun. Heat Mass Transf. 2010, 37, 687-694. [CrossRef]

15. Sajid, M.U.; Ali, H.M. Recent advances in application of nanofluids in heat transfer devices: A critical review. Renew. Sustain. Energy Rev. 2019, 103, 556-592. [CrossRef]

16. Mahmoudi, A.H.; Shahi, M.; Raouf, A.H.; Ghasemian, A. Numerical study of natural convection cooling of horizontal heat source mounted in a square cavity filled with nanofluid. Int. Commun. Heat Mass Transf. 2010, 37, 1135-1141. [CrossRef]

17. Reza-E-Rabbi, S.; Ahmmed, S.F.; Arifuzzaman, S.; Sarkar, T.; Khan, S. Computational modelling of multiphase fluid flow behaviour over a stretching sheet in the presence of nanoparticles. Eng. Sci. Technol. Int. J. 2020, 23, 605-617. [CrossRef]

18. Raisi, A. The effect of conductive baffles on natural convection in a power-law fluid-filled square cavity. J. Braz. Soc. Mech. Sci. Eng. 2018, 40, 33. [CrossRef]

19. Armaghani, T.; Kasaeipoor, A.; Izadi, M.; Pop, I. MHD natural convection and entropy analysis of a nanofluid inside T-shaped baffled enclosure. Int. J. Numer. Methods Heat Fluid Flow 2018, 28, 2916-2941. [CrossRef]

20. Khorasanizadeh, H.; Amani, J.; Nikfar, M. Numerical investigation of Cu-water nanofluid natural convection and GOE within a cavity with an embedded conductive baffle. Sci. Iran. 2012, 19, 1996-2003. [CrossRef]

21. Kandaswamy, P.; Lee, J.; Hakeem, A.A.; Saravanan, S. Effect of baffle-cavity ratios on buoyancy convection in a cavity with mutually orthogonal heated baffles. Int. J. Heat Mass Transf. 2008, 51, 1830-1837. [CrossRef] 
22. Asl, A.K.; Hossainpour, S.; Rashidi, M.M.; Sheremet, M.; Yang, Z. Comprehensive investigation of solid and porous fins influence on natural convection in an inclined rectangular enclosure. Int. J. Heat Mass Transf. 2019, 133, 729-744. [CrossRef]

23. Khanafer, K.; AlAmiri, A.; Bull, J. Laminar natural convection heat transfer in a differentially heated cavity with a thin porous fin attached to the hot wall. Int. J. Heat Mass Transf. 2015, 87, 59-70. [CrossRef]

24. Liu, Y.; Lei, C.; Patterson, J.C. Natural convection in a differentially heated cavity with two horizontal adiabatic fins on the sidewalls. Int. J. Heat Mass Transf. 2014, 72, 23-36. [CrossRef]

25. Alnaqi, A.A.; Aghakhani, S.; Pordanjani, A.H.; Bakhtiari, R.; Asadi, A.; Tran, M.-D. Effects of magnetic field on the convective HTR and GOE of a nanofluid in an inclined square cavity equipped with a conductor fin: Considering the radiation effect. Int. J. Heat Mass Transf. 2019, 133, 256-267. [CrossRef]

26. Al-Rashed, A.A. Investigating the effect of alumina nanoparticles on heat transfer and GOE inside a square enclosure equipped with two inclined blades under magnetic field. Int. J. Mech. Sci. 2019, 152, 312-328. [CrossRef]

27. Aghakhani, S.; Ghasemi, B.; Pordanjani, A.H.; Wongwises, S.; Afrand, M. Effect of replacing nanofluid instead of water on heat transfer in a channel with extended surfaces under a magnetic field. Int. J. Numer. Methods Heat Fluid Flow 2019, 29, 1249-1271. [CrossRef]

28. Yan, S.-R.; Aghakhani, S.; Karimipour, A. Influence of a membrane on nanofluid heat transfer and irreversibilities inside a cavity with two constant-temperature semicircular sources on the lower wall: Applicable to solar collectors. Phys. Scr. 2020, 95, 085702. [CrossRef]

29. Tian, M.-W.; Rostami, S.; Aghakhani, S.; Goldanlou, A.S.; Qi, C. A techno-economic investigation of 2D and 3D configurations of fins and their effects on heat sink efficiency of MHD hybrid nanofluid with slip and non-slip flow. Int. J. Mech. Sci. 2021, 189, 105975. [CrossRef]

30. Selimefendigil, F.; Öztop, H.F. Corrugated conductive partition effects on MHD free convection of CNT-water nanofluid in a cavity. Int. J. Heat Mass Transf. 2019, 129, 265-277. [CrossRef]

31. Sajjadi, H.; Delouei, A.A.; Sheikholeslami, M.; Atashafrooz, M.; Succi, S. Simulation of three dimensional MHD natural convection using double MRT Lattice Boltzmann method. Phys. A Stat. Mech. Its Appl. 2019, 515, 474-496. [CrossRef]

32. Ellahi, R.; Zeeshan, A.; Shehzad, N.; Alamri, S.Z. Structural impact of kerosene- $\mathrm{Al}_{2} \mathrm{O}_{3}$ nanoliquid on MHD Poiseuille flow with variable thermal conductivity: Application of cooling process. J. Mol. Liq. 2018, 264, 607-615. [CrossRef]

33. Rashad, A.; Armaghani, T.; Chamkha, A.; Mansour, M. GOE and MHD natural convection of a nanofluid in an inclined square porous cavity: Effects of a heat sink and source size and location. Chin. J. Phys. 2018, 56, 193-211. [CrossRef]

34. Sheikholeslami, M.; Sadoughi, M. Mesoscopic method for MHD nanofluid flow inside a porous cavity considering various shapes of nanoparticles. Int. J. Heat Mass Transf. 2017, 113, 106-114. [CrossRef]

35. Arifuzzaman, S.; Khan, S.; Al-Mamun, A.; Reza-E-Rabbi, S.; Biswas, P.; Karim, I. Hydrodynamic stability and heat and mass transfer flow analysis of MHD radiative fourth-grade fluid through porous plate with chemical reaction. J. King Saud Univ. Sci. 2019, 31, 1388-1398. [CrossRef]

36. Aghakhani, S.; Pordanjani, A.H.; Afrand, M.; Sharifpur, M.; Meyer, J.P. Natural convective heat transfer and entropy generation of alumina/water nanofluid in a tilted enclosure with an elliptic constant temperature: Applying magnetic field and radiation effects. Int. J. Mech. Sci. 2020, 174, 105470. [CrossRef]

37. Mahian, O.; Mahmud, S.; Pop, I. Analysis of first and second laws of thermodynamics between two isothermal cylinders with relative rotation in the presence of MHD flow. Int. J. Heat Mass Transf. 2012, 55, 4808-4816. [CrossRef]

38. Pordanjani, A.H.; Jahanbakhshi, A.; Nadooshan, A.A.; Afrand, M. Effect of two isothermal obstacles on the natural convection of nanofluid in the presence of magnetic field inside an enclosure with sinusoidal wall temperature distribution. Int. J. Heat Mass Transf. 2018, 121, 565-578. [CrossRef]

39. Pordanjani, A.H.; Aghakhani, S. Numerical Investigation of Natural Convection and Irreversibilities between Two Inclined Concentric Cylinders in Presence of Uniform Magnetic Field and Radiation. Heat Transf. Eng. 2021, 1-21. [CrossRef]

40. Salari, M.; Kasaeipoor, A.; Malekshah, E.H. Three-dimensional natural convection and entropy generation in tall rectangular enclosures filled with stratified nanofluid/air fluids. Heat Transf. Res. 2018, 49, 685-702. [CrossRef]

41. Al-Zamily, A.M.J. Analysis of natural convection and GOE in a cavity filled with multi-layers of porous medium and nanofluid with a heat generation. Int. J. Heat Mass Transf. 2017, 106, 1218-1231. [CrossRef]

42. Chamkha, A.; Ismael, M.; Kasaeipoor, A.; Armaghani, T. GOE and Natural Convection of CuO-Water Nanofluid in C-Shaped Cavity under Magnetic Field. Entropy 2016, 18, 50. [CrossRef]

43. Ali, M.M.; Akhter, R.; Alim, A. MHD natural convection and entropy generation in a grooved enclosure filled with nanofluid using two-component non-homogeneous model. SN Appl. Sci. 2020, 2, 1-25. [CrossRef]

44. Li, Z.; Hussein, A.K.; Younis, O.; Afrand, M.; Feng, S. Natural convection and entropy generation of a nanofluid around a circular baffle inside an inclined square cavity under thermal radiation and magnetic field effects. Int. Commun. Heat Mass Transf. 2020, 116, 104650. [CrossRef]

45. Acharya, S.; Dash, S.K. Natural Convection and Entropy Generation in a Porous Enclosure Filled with Non-Newtonian Nanofluid. J. Thermophys. Heat Transf. 2021, 35, 438-458. [CrossRef]

46. Zhou, X.; Jiang, Y.; Li, X.; Cheng, K.; Huai, X.; Zhang, X.; Huang, H. Numerical investigation of heat transfer enhancement and GOE of natural convection in a cavity containing nano liquid-metal fluid. Int. Commun. Heat Mass Transf. 2019, 106, 46-54. [CrossRef] 
47. Dutta, S.; Goswami, N.; Biswas, A.K.; Pati, S. Numerical investigation of magnetohydrodynamic natural convection heat transfer and GOE in a rhombic enclosure filled with Cu-water nanofluid. Int. J. Heat Mass Transf. 2019, 136, 777-798. [CrossRef]

48. Vajjha, R.S.; Das, D.K. Experimental determination of thermal conductivity of three nanofluids and development of new correlations. Int. J. Heat Mass Transf. 2009, 52, 4675-4682. [CrossRef]

49. Hassani, S.; Saidur, R.; Mekhilef, S.; Hepbasli, A. A new correlation for predicting the thermal conductivity of nanofluids; using dimensional analysis. Int. J. Heat Mass Transf. 2015, 90, 121-130. [CrossRef]

50. Maxwell, J.C.; Thompson, J.J. A Treatise on Electricity and Magnetism; Clarendon Press: Oxford, UK, 1904.

51. Brinkman, H.C. The Viscosity of Concentrated Suspensions and Solutions. J. Chem. Phys. 1952, 20, 571-581. [CrossRef]

52. Oztop, H.F.; Abu-Nada, E. Numerical study of natural convection in partially heated rectangular enclosures filled with nanofluids. Int. J. Heat Fluid Flow 2008, 29, 1326-1336. [CrossRef]

53. Aminossadati, S.; Ghasemi, B. Natural convection cooling of a localised heat source at the bottom of a nanofluid-filled enclosure. Eur. J. Mech. B Fluids 2009, 28, 630-640. [CrossRef] 